\title{
Transcriptional and metabolic profiling reveals modulation of genes associated to drought tolerance by chloroplast redox poise in potato
}

\author{
Juan Pierella Karlusich ${ }^{1}$, Rocío Arce ${ }^{2}$, Fahimeh Shahinnia ${ }^{3}$, Sophia Sonnewald ${ }^{4}$, Uwe \\ Sonnewald $^{5}$, Matias Zurbriggen ${ }^{6}$, Mohammad-Reza Hajirezaei ${ }^{7}$, and Carrillo Nestor ${ }^{2}$ \\ ${ }^{1}$ Ecole Normale Supérieure Département de Biologie \\ ${ }^{2}$ Instituto de Biología Molecular y Celular de Rosario \\ ${ }^{3}$ Institute of Plant Genetics and Crop Plant Reseacrh \\ ${ }^{4}$ Friedrich-Alexander-University Erlangen-Nürenberg \\ ${ }^{5}$ Friedrich-Alexander Universität Erlangen-Nürnberg \\ ${ }^{6}$ Institute of Synthetic Biology and Cluster of Excellence on Plant Sciences (CEPLAS), \\ University of Düsseldorf, Düsseldorf, Germany \\ ${ }^{7}$ Institute of Plant Genetics and Crop Plant Research
}

May 6, 2020

\begin{abstract}
Water stress causes considerable yield loss in potato, usually considered a drought-sensitive crop. Photosynthesis is a primary target of abiotic stresses, and manipulation of photosynthetic electron distribution in chloroplasts by introducing the alternative electron carrier flavodoxin increased tolerance of model plants to multiple environmental challenges including drought, suggesting that a similar strategy could function in crops. We show herein that expression of a plastid-targeted cyanobacterial flavodoxin in potato plants protected photosynthetic activities even at a pre-symptomatic stage of drought stress. Transcriptional profiling revealed that $5-6 \%$ of leaf-expressed genes were regulated by flavodoxin in the absence of stress, representing pathways modulated by chloroplast redox status during normal growth. The majority $(68.5 \%)$ of drought-responsive genes were also affected by flavodoxin, whose main effect was to mitigate changes in gene expression caused by water deficit, suggesting that flavodoxinexpressing plants suffered less stress than their wild-type siblings, and accordingly displayed an attenuated response to the adverse condition. Expression of 290 drought-responsive genes was primed in the transformants in the absence of stress, and could contribute to drought tolerance. Yield improvement under chronic water limitation was moderate but significant in flavodoxin-expressing plants, indicating that the flavoprotein has potential to improve major agronomic traits in potato.
\end{abstract}

\section{INTRODUCTION}

Environmental stress represents the most important single factor limiting crop yield worldwide, a situation that will certainly aggravate in the near future as a consequence of global climate changes (IPCC 2014; Buchanan, B.B., Gruissem, W. \& Jones 2015). Among the adverse conditions that constrain plant growth and reproduction, drought has the highest impact in quantitative terms (FAO 2014). Projections of increased desertification in several Mediterranean-type environments of Europe, Australia, South America and subSaharan Africa are consistent across simulation models (IPCC 2014). Water restriction negatively affects photosynthetic rates by decreasing $\mathrm{CO}_{2}$ availability as a result of stomatal closure, and by feedback inhibition due to limitations in photosynthate transport to sink organs (Romero, Alarcón, Valbuena \& Galeano 2017; Urban, Aarrouf \& Bidel 2017). Many changes associated to water deficit are therefore detected in the leaves 
and accordingly, drought responses are inextricably linked to photosynthesis and chloroplast biochemistry (Zingaretti, Inacio, de Matos Pereira, Paz \& de Castro Franca 2013).

Potato (Solanum tuberosum L.) is the third most important food crop in the world (Visser et al. 2009; FAO 2014), and is vulnerable to drought, salinity and other environmental stresses, which affect tuber yield and quality (Vasquez-Robinet et al. 2008; Muñiz García, Cortelezzi, Fumagalli \& Capiati 2018). The situation is particularly critical in developing countries where potato is most important as an affordable and nutritionally rich food supply (Romeroet al. 2017), and where the impact of global climate change is predicted to be more severe (IPCC 2014; Muñiz García et al.2018). Therefore, breeders face increasing pressure to develop new lines with improved drought tolerance while keeping high crop yield, tuber quality and market acceptance (Romero et al. 2017).

Water limitation elicits a very complex plant response, which combines physiological, cellular and metabolic adaptations to the stress situation, and involves genome-wide changes in gene expression patterns. While many drought-responsive genes have been identified, it is presently difficult to define the role played by most of them in the tolerance against this environmental challenge (André et al.2009). As most other abiotic stresses, water deficit causes a rise of reactive oxygen species (ROS) levels, especially in leaves (Gómez, Vicino, Carrillo \& Lodeyro 2019). In turn, the drought response of genes involved in ROS metabolism depends on the plant cultivar, its degree of tolerance and the duration and intensity of the stress treatment (Cruz De Carvalho 2008). Vasquez-Robinet et al. (2008) found that the higher drought tolerance displayed by Andean potato genotypes was related to enhanced expression of genes encoding antioxidant proteins located in chloroplasts. Moreover, potato transformation with genes related to ROS scavenging led to lines exhibiting improved performance under water deprivation (Ahmad et al. 2010; Eltayebet al. 2011; Cheng, Deng, Kwak, Chen \& Eneji 2013), indicating that manipulation of ROS metabolism is a promising strategy to improve drought tolerance (Gómez et al. 2019).

We have generated tobacco plants with increased tolerance to multiple sources of abiotic stress by introducing a cyanobacterial flavodoxin (Fld) directed to chloroplasts (Tognetti et al. 2006; Zurbriggenet al. 2008). Fld is an electron carrier flavoprotein present in bacteria and some algae that displays essentially the same activities and redox interactions as the iron-sulfur protein ferredoxin (Fd), including functional integration as a final electron acceptor in the photosynthetic electron transport chain (PETC). Fld expression is normally induced in microorganisms under conditions of iron starvation and environmental stress that cause Fd downregulation, taking over Fd functions as its levels decline, and preventing over-reduction of the PETC and ROS propagation (Zurbriggen et al. 2008; Pierella Karlusich, Lodeyro \& Carrillo 2014). The Fld gene is absent from plant genomes (Pierella Karlusich, Ceccoli, Graña, Romero \& Carrillo 2015), but introduction of a plastid-targeted Fld improved delivery of reducing equivalents to productive pathways of the chloroplast, which in turn restricted plastid ROS production and increased tolerance to drought and other stresses (Tognetti et al. 2006, 2007; Zurbriggen et al. 2008; Li et al. 2017).

The need for drought-tolerant potato lines prompted us to evaluate the Fld approach in this crop. e describe herein the preparation and characterization of potato plants expressing a plastid-localized Fld, and displaying improved photosynthesis, growth and tuber yield under conditions of water deprivation. To gain further insights into the mechanism(s) of Fld-associated stress tolerance, we generated genome-wide transcript profiles from wild-type (WT) and Fld-expressing potato leaves at a pre-symptomatic stage of water restriction to evaluate early responses to hydric stress, and combined this approach with metabolic profiling of carbohydrates and amino acids. The results provide a detailed snapshot of how chloroplast redox biochemistry affects gene expression metabolism in a major crop during this agronomically relevant abiotic stress.

\section{MATERIALS AND METHODS}

\subsection{Preparation and growth of potato plants expressing cyanobacterial Fld}

Construction of the pCAMBIA2200 vector encoding plastid-targeted Fld from Anabaena PCC7119 ( $p$ fld ) has been described elsewhere (Tognetti et al. 2006). Briefly, a DNA sequence encoding the chloroplast transit peptide of pea ferredoxin-NADP ${ }^{+}$reductase (FNR) was fused in-frame to the 5 '-end of the Anabae- 
$n a \mathrm{PCC} 7119$ fld gene and placed under control of the cauliflower mosaic virus (CaMV) 35S promoter to allow for constitutive expression and plastid targeting in transformed plants (Supplementary Figure S1a). Potato plants (S. tuberosum cv Solara) were transformed by agroinfiltration (Rocha-Sosa et al., 1989) to generate Stpfldlines (for $\boldsymbol{S}$. tuberosum p lastidic Fld, Supplementary Figure S1a). The presence of Fld in cleared leaf extracts was evaluated by sodium dodecyl sulfate-polyacrylamide gel electrophoresis (SDS-PAGE) and immunoblot detection with specific antisera (Mayta et al. 2019). Plants were propagated in vitro according to Rocha-Sosa et al. (1989), transferred to soil, and grown at $400 \mu \mathrm{mol}$ photons $\mathrm{m}^{-2} \mathrm{~s}^{-1}$, a 16 -h photoperiod, $25 / 22^{\circ} \mathrm{C}$ and a relative humidity of $80 \%$ (growth chamber conditions).

For short-term drought assays, tuber slices of similar sizes corresponding to the various lines were planted in 3 -L soil pots. Plants were watered to $100 \%$ field capacity (FiC) for 30 days and then subjected to drought by water withdrawal under growth chamber conditions.

For long-term drought treatments, tuber slices of WT andStpfld 252 lines were planted in 15-L soil pots, watered to $100 \% \mathrm{FiC}$ for the first 30 days of growth. By that time, plants were beginning to set tubers. Watering was interrupted until the soil reached $40 \% \mathrm{FiC}$ and watered again to $70 \% \mathrm{FiC}$. This procedure was repeated until plants were harvested, 120 days post germination (Supplementary Figure S2).

\subsection{Relative water content determinations}

Young fully expanded leaves from the fifth node and comparable in size were sampled for relative water content (RWC) determinations. Leaves were weighed immediately after sampling (fresh weight), floated in the dark for $48 \mathrm{~h}$ to achieve maximal turgidity (saturated weight), oven-dried $\left(80^{\circ} \mathrm{C}\right)$ for $16 \mathrm{~h}$ and weighed again (dry weight). RWC was calculated according to the formula: [(fresh weight - dry weight)/(saturated weight - dry weight)] x 100. Three biological replicates were assayed for each treatment.

\subsection{Photosynthetic measurements}

Chlorophyll $a$ fluorescence determinations were performed using a MultispeQ-Beta device controlled by the PhotosynQ platform software (Kuhlgert et al. 2016). Measurements were carried out at midday on leaves from the fourth and fifth nodes of 5 independent plants per line, and photosynthetic parameters were calculated according to Baker (2008).

\subsection{In situ detection of reactive oxygen species}

ROS cellular localization was determined by confocal microscopy in an Eclipse TE-2000-E2 Confocal Laser Scanning Microscope (Nikon), essentially as described by Mayta et al. (2018). Discs (110 $\mathrm{mm}^{2}$ in diameter) were collected during the light period from leaves belonging to the fourth node of 5 different plants per line, vacuum-infiltrated in the dark with $50 \mu \mathrm{M}$ DCFDA in $10 \mathrm{mM}$ Tris-HCl pH 7.5 and $0.1 \%$ (v/v) Tween-20, incubated in the dark for $1 \mathrm{~h}$ in the same solution, washed briefly and mounted in water. Imaging was performed by scanning 5 optical slices (with an interval of $1 \mu \mathrm{m}$ ) of the palisade parenchyma immediately below the epidermis. Fluorescence intensities were estimated using Fiji software (Schindelin et al.2012), using the z-projections of the different stacks.

\subsection{RNA isolation, cDNA labeling and microarray hybridization}

For microarray analysis, 30-days old WT and Stpfld 252 plants were subjected to short-term drought treatment as described above. Young fully expanded leaves belonging to the fifth node were collected after three days of water withdrawal in the treated group. Leaf material from 10 different plants of each genotype and treatment were frozen in liquid nitrogen and ground with Mixer Mill MM400 (Retsch). Two pools of biological samples, each from two independent experiments per genotype and condition were used for the microarray analysis. Leaf RNA was extracted according to Logemann et al. (1987). RNA quantity and quality were determined with a NanoDrop spectrophotometer (Thermo Scientific, Wilmington, DE) and by visual inspection after electrophoresis, respectively. One microgram of RNA from each sample was treated with RQ1 DNase (Promega, Madison, WI) according to the manufacturer's instructions, and used as template to generate cDNA with M-MLV Reverse Transcriptase (Promega) and oligo (dT)12-18. 
Gene expression profiles were assessed with Potato Oligo Chip Initiative (POCI) microarray, a 60-mer oligobased $4 \times 44 \mathrm{k}$ Agilent microarray (AMADID: 015425), consisting of 42034 60-mer probes (Kloostermanet al. 2008). Sample labeling and hybridization were performed as described in the one-color microarray-based gene expression analysis protocol including the one-color RNA spike-in Kit (v5.0.1, Agilent Technologies). Slides were scanned with an Agilent microarray scanner (G2505B) at high resolution. Data were extracted using feature extraction software (v9.5.3, Agilent) by a standard protocol.

\subsection{Microarray data analysis}

Data processing and statistical analysis were carried out with the Bioconductor library limma (Ritchie et al. 2015). Background correction and normalization were performed using the "normexp" and quantile methods, respectively. We only considered probes whose intensity was more than $10 \%$ above background on at least one genotype/treatment combination. An empirical Bayes method with moderated $t$-statistic was employed for the determination of the genes with statistically significant changes, whereas the Benjamini and Hochberg's method was used to control false discovery rates (FDR). Differentially expressed (DE) genes were identified from pairwise comparisons when FDR $<0.05$ and fold-change (FC) was $>2$ or $<0.5$. Pathway over-representation analyses between lines or treatment comparisons were performed with PageMan (Usadel et al.2006) using Fisher's exact test with Bonferroni correction (FDR $<0.05)$.

Based on the results of the multiple comparison test described above, genes were defined as induced (FC $>2$ and FDR $<0.05$ ), repressed $(\mathrm{FC}<0.5$ and FDR $<0.05$ ) or unaffected in each of the four pairwise comparison combinations (Stpfld 252 vs . WT under control conditions, Stpfld $252 \mathrm{vs}$. WT under drought, drought vs . control in Stpfld 252 line, drought vs. control in WT line). An ad hoc -made R script was used to group the genes sharing the same results in the four pairwise comparisons, and thus defining the same cluster. Graphics representing the resulting clusters were prepared with the R library ggplot2 (Wickham 2009). Pathway over-representation in each cluster was determined as described above. Mapman ontology was used for functional annotation (Thimm et al. 2004) employing a mapping file updated in May 2018 (stu_Agilent_4x44k_2018-05-25_mapping.txt) from the GoMapMan website resource (www.gomapman.org; Ramšaket al. 2014).

\subsection{Validation of DE genes by quantitative reverse transcription (qRT)-PCR}

For qRT-PCR analysis, drought treatment, plant age and sampled leaves were the same as in the microarray experiments. Total RNA was extracted from leaf tissue using the TriPure reagent (Sigma-Aldrich), according to the manufacturer's instructions, and reverse-transcribed with the M-MLV enzyme (Invitrogen) as indicated before. The qRT-PCR reactions were carried out in a Master cycler Rep realplex ${ }^{4}$ thermocycler (Eppendorf) using Platinum Taq DNA polymerase (Invitrogen) and SYBR Green I (Roche) to monitor the synthesis of double-stranded DNA under the following conditions: $95^{\circ} \mathrm{C}$ for 2 min and then 40 cycles of $95^{\circ} \mathrm{C}$ for 15 $\mathrm{s}, 55^{\circ} \mathrm{C}$ for $30 \mathrm{~s}$, and $72^{\circ} \mathrm{C}$ for $40 \mathrm{~s}$. The relative abundance of transcripts was estimated with the $\Delta \Delta \mathrm{Ct}$ method (Schmittgen \& Livak 2008), and normalized to the gene encoding elongation factor-1 $\alpha$ (Genbank accession number AB061263.1 ). Primers used in this study are listed in Supplementary Table S1. Each qRT-PCR reaction set included 5-6 biological and 2 technical replicate samples, and water used as a negative no-template control instead of cDNA.

\subsection{Metabolic profiling}

Soluble sugars and soluble amino acids were determined essentially as described by Ghaffari et al. (2016). Numerical analysis and quantification of individual compounds were carried out using Empower Pro software (Waters, Milford, MA) and authentic standards; respectively.

\subsection{Availability of Supporting Data}

Data reported in this publication have been deposited in NCBI's Gene Expression Omnibus (Edgar, Domrachev \& Lash 2002) and are accessible through GEO Series accession number GSE149503 (https://www.ncbi.nlm.nih.gov/geo/query/acc.cgi?acc=GSE149503). 


\section{Statistical analyses}

The determinations of photosynthetic activity, RWC, fluorescence intensity, qRT-PCR and amino acid levels were analyzed using one-way ANOVA and Tukey's Multiple Comparison Test, whereas measurements of fresh weight $(\mathrm{FW})$, water potential and tuber yield were analyzed with the non-parametric Mann-Whitney test. Statistical significance was specified in each experiment.

\section{RESULTS}

\subsection{Expression of plastid-targeted Fld improved potato drought tolerance}

Potato plants were transformed with the fld -containing plasmid described by Tognetti et al. (2006) using published procedures (Rocha-Sosa et al. 1989; see Materials and Methods). Several independent Stpfld lines were obtained, expressing different Fld levels as revealed by SDS-PAGE and immunoblotting (Supplementary Figure S1b). The flavoprotein was largely recovered from leaf extracts as a mature-sized product (Supplementary Figure S1b), suggesting plastid import and processing (Tognetti et al. 2006). Traces of Fld precursor and processing intermediates were detected in highly expressing Stpfld 252 and Stpfld 239 lines (Supplementary Figure S1b), as already observed in tobacco (Tognetti et al.2006).

To evaluate the drought tolerance conferred by Fld introduction, 30-days old WT and Stpfld plants cultured under growth chamber conditions (see Materials and Methods) were exposed to hydric stress by interrupting irrigation. Visual symptoms of stress were observed in WT leaves after $\sim 9$ days of water withdrawal, and by 14 days wilting extended to both leaves and stems (Figure 1a), correlating with significant decreases in leaf water contents (Supplementary Figure S3). Under the same conditions, Fld-expressing plants looked healthy (Figure 1a) and retained leaf turgor (Supplementary Figure S3).

As indicated, photosynthesis is one of the most sensitive targets of water deficit (Urban et al. 2017). Measurements of chlorophyll $a$ fluorescence on WT leaves revealed a fast decrease in the maximum quantum efficiency of photosystem II (PSII), as represented by the $F_{\mathrm{v}}{ }^{\prime} / F$ m' ratio, evident after only 2-3 days of stress (Figure 1b). This parameter is customarily used to monitor photodamage to PSII (Baker $2008)$. The quantum yield of PSII $\left(\Phi_{\mathrm{PSII}}\right)$, which provides an estimation of electron flow through this photosystem, also declined steadily with the days of treatment (Figure 1c). Finally, dissipation of the excess of energy that cannot be used for photochemistry, a process monitored by the non-photochemical quenching of chlorophyll fluorescence (NPQt), increased as stress became more severe (Figure 1d). The results indicate that photosynthetic impairment caused by the drought regime preceded visible tissue dehydration and increased as wilting progressed (Figure 1b-d). These detrimental effects were largely prevented by Fld presence in chloroplasts of the transgenic plants, as indicated by differential preservation of PSII integrity and electron flow, and comparatively lower values of NPQt (Figure 1b-d). Only minor differences, without statistical significance, were observed in well-watered plants of all lines during the timespan of the assay (Figure 1b-d).

ROS build-up is also a common feature of environmental stress conditions (Czarnocka \& Karpiński 2018; Gómez et al. 2019), and the protective effect of Fld has been linked to its role as a general antioxidant specific for chloroplasts (Zurbriggen et al. 2008; Pierella Karlusich et al. 2014; Rossi et al. 2017). To visualize ROS accumulation in water-deprived plants, leaves were infiltrated with the ROS-sensitive fluorescent probe 2',7'dichlorodihydrofluorescein diacetate (DCFDA) for detection by confocal laser scanning microscopy (Materials and Methods). Results are illustrated in Figure 2 for leaves of Stpfld 252 plants, a line displaying high levels of Fld expression (Supplementary Figure 1b) and drought tolerance (Figure 1). Under the conditions employed, DCFDA fluorescence was nearly undetectable in watered plants from both genotypes (Figure 2a). At 14 days of water withdrawal, WT leaves showed significant increases in ROS-associated fluorescence (Figure 2b). Actually, ROS build-up was already evident after only 3 days of treatment, indicating that increased production of these reactive species was an early manifestation of the plant stress response (Figure 2b). Presence of plastid Fld largely prevented this rise (Figure 2b). About $60 \%$ of total ROS was associated to chloroplasts in both conditions and genotypes (Figure 2c).

\subsection{Microarray analysis of drought-stressed plants expressing chloroplast Fld}


Transcriptional profiling is a powerful experimental approach to investigate plant responses to environmental challenges. Most transcriptome analyses on water-restricted potatoes have been performed on plants showing stress symptoms (Evers et al. 2010; Kondrak, Marincs, Antal, Juhasz \& Banfalvi 2012; Zhang et al. 2014). Fld provided protection against drought effects occurring at a pre-symptomatic stage that might contribute to the differential tolerance exhibited by Fld-expressing plants. Therefore, a genome-wide transcriptional profiling was carried out using WT and Stpfld 252 leaves sampled at 3 days of treatment as RNA source, together with their corresponding watered controls. Once again, the Stpfld 252 line was chosen because of its high levels of Fld accumulation and drought protection.

Gene expression profiles were determined using a single-channel Agilent potato microarray consisting of 42034 probes. We did not carry out any filtering for potential redundant probes mapping the same transcript due to the difficulties related to the highly heterozygous nature of potato genomes, their polyploidy variance, the different accessions used the microarray design, the reference genome sequencing and our current work. Functional annotation was based on the Mapman ontology using the GoMapman website resource (Ramšak et al. 2014). More than $80 \%$ of the genes included in the microarray could be assigned to a functional category. The total number of leaf-expressed genes that passed the background correction and filtering processes (Materials and Methods) was similar for the two genotypes under control conditions and represented about $75 \%$ of the total genes present in the microarray.

\subsection{Chloroplast Fld affected gene expression patterns of potato leaves in the absence of stress}

We first evaluated the effect of chloroplast Fld on gene expression in leaves of potato plants grown under normal conditions. Analysis of transcript levels in WT and Stpfld 252 leaves showed that 1097 genes were induced 2-fold or more by Fld relative to WT siblings, with 181 increasing more than 4-fold in their expression. Also, 578 transcripts declined to $50 \%$ or less in Stpfld 252 leaves compared to the wild type, 54 of which accumulated below or equal to 25\% (Figure 3, Supplementary Table S2). Functional enrichment analysis of these DE transcripts showed that among the genes induced in Stpfld 252 plants there was a remarkable over-representation of those associated to protein degradation by the proteasome (Figure 3, Supplementary Table S2). It is worth noting that a similar induction pattern has been observed in Fld-expressing tobacco plants (Pierella Karlusich et al. 2017), indicating that plastid-located Fld exerts a key regulatory role in the accumulation of proteasomal components in different species, presumably through modulation of chloroplast redox chemistry.

Functional categories that displayed differential repression by Fld comprised trehalose synthesis, nitrate metabolism, amino acid degradation and traits associated to metal uptake and utilization such as $\mathrm{Cd}$ - and $\mathrm{Al}-$ induced proteins, $\mathrm{Cu}$ chaperones and Fe-chelate reductases. Noteworthy, ethylene metabolism and signaling were consistently down-regulated in Stpfld 252 plants, including 29 genes encoding transcription factors of the apetala2/ethylene-responsive family (Figure 3, Supplementary Table S2). These observations also agree with earlier reports on Fld-expressing tobacco plants (Pierella Karlusich et al. 2017). The mechanisms underlying this repression are unknown and deserve further investigation.

\subsection{Water deprivation caused extensive transcriptional reprogramming in both WT and Fld- expressing leaves}

Gene expression levels were determined after 3 days of water restriction and transcripts displaying 2-fold or higher differences compared to control well-watered conditions were classified as DE. Distribution of these transcripts between treatments and genotypes (depicted as Venn diagrams in Figure 4) indicates that 999 genes were induced by drought in both lines, 1530 genes only in the non-transformed plants, and 698 uniquely in Stpfld 252 leaves $(\mathrm{FC}>2, \mathrm{FDR}<0.05)$. In contrast, 1505 genes were repressed by drought in both lines, 1667 only in the wild type and 895 exclusively inStpfld 252 plants. In summary, drought induced 2529 genes in WT plants against 1697 in Stpfld 252 siblings, and repressed 3172 genes in the wild type versus 2400 in the transformant. Hence, the overall effect of Fld presence was to mitigate the changes in gene expression driven by the drought treatment; either induction or repression.

Functional enrichment analysis identified pathways and functional categories that were significantly affected 
in response to water withdrawal in the two genotypes. Among the categories that showed drought-dependent induction in both WT and Stpfld 252 plants, abiotic stress was over-represented. The opposite situation,e.g. , pathways repressed by water limitation in the two lines, is exemplified by cell wall metabolism and kinasedependent protein modification and signaling (Figure 4, Supplementary Table S3).

Several DE genes showed differential stress responses in only one genotype. Drought-dependent inactivation of photosynthesis in WT plants (Figure 1b-d) was paralleled at the transcript level by widespread downregulation of genes encoding photosynthetic components. Repression particularly impaired light reactions, and was largely prevented by chloroplast Fld (Figure 4, Supplementary Table S3), correlating with the protection of photosynthetic activities in the transformant (Figure 1b-d).

While the effect of drought on leaf gene expression was predominantly repressive (Figure 4, Supplementary Table S3), stress-dependent induction was also important in the wild type with more than 2500 genes affected. Enriched functional categories included protein metabolism with 98 DE genes (mostly ribosomal proteins), biotic stress and amino acid synthesis (Figure 4, Supplementary Table S3). Differential induction of all these pathways was totally or partially abolished by Fld expression in chloroplasts, suggesting once again that the water deficit regime had less impact in the transgenic plants than in their WT siblings.

Finally, some functional categories were specifically over-represented in the transformant, including droughtassociated up-regulation of membrane transport, sulfur assimilation and flavonoid synthesis (Figure 4, Supplementary Table S3).

\subsection{Expression of one-third of drought-responsive genes was not affected by Fld presence}

Clustering of DE transcripts allows identification of gene groups that display similar expression patterns and presumably share common regulatory pathways. Total leaf transcripts detected in our microarray were thus grouped into 47 clusters of widely different size. The most highly populated one (27622 members, corresponding to $\sim 5 \%$ of all leaf-expressed genes) included those transcripts whose levels were not affected by treatment or genotype. Of the remaining 46 clusters containing genes differentially expressed in response to water limitation and/or Fld presence (4847 DE genes), we focused in 17 clusters that were either highly populated or enriched in pathways and functional categories associated to stress responses, redox biochemistry and chloroplast functions, comparing vis-à-vis those that exhibit contrasting expression behaviors (e.g ., induction vs. repression). Distribution of DE transcripts in these 17 clusters can be found in Supplementary Tables S4-9, and the responses represented by the other 29 clusters are described in Supplementary Figure S4.

Clusters comprising genes that display a similar drought response in the presence or absence of Fld represent $31.5 \%$ of total DE transcripts, of which 601 were induced (cluster 1) and 929 were repressed (cluster 2). Cluster 1 was enriched in genes coding for components of the cytosolic branch of glycolysis, ethylene synthesis, heat stress and signal transduction associated to abscisic acid (Supplementary Figure S5; Supplementary Table S4). Cluster 2 exhibited a remarkable over-representation of genes belonging to cell wall metabolism such as cellulose synthases, cellulases and pectinesterases, which declined in both lines under water deficit (Supplementary Figure S5; Supplementary Table S4). Several receptor kinases of the leucine-rich repeat family were also repressed by drought irrespective of genotype.

\subsection{Fld-regulated genes whose expression was unaffected by drought}

Of the 1675 genes whose expression was modified by Fld presence under normal growth conditions (Figure 3; Supplementary Table S2), 1336 ( $~ 80 \%)$ were also affected by water limitation, and distributed among various clusters described below. The remaining 339 genes responded solely to Fld presence and were grouped in clusters 3 and 4 (Supplementary Figure S6, Supplementary Table S5). Most of these Fld-responsive genes were induced, constituting cluster 3 with 221 members and conspicuous over-representation of the ubiquitin (Ub)proteasome system. Cluster 4 contained 88 genes repressed by the flavoprotein, with amino acid degradation, metal handling and cytokinin signal transduction representing enriched functional categories (Supplementary Figure S6; Supplementary Table S5). 


\subsection{Genes whose drought response was ameliorated by chloroplast Fld}

A significant number of genes showed different expression patterns under water stress conditions depending on the presence of Fld, exhibiting lower induction (clusters 5 and 6) or repression (clusters 7 and 8) inStpfld 252 leaves relative to WT counterparts (Figure 5, Supplementary Table S6). These four clusters comprise $33 \%$ of total DE transcripts (1611 genes), and collectively represent the most common response to treatment and genotype. Cluster 5 contained 546 genes whose drought-dependent induction was completely abolished by Fld, while for 274 genes of cluster 6 the effect of Fld was only partial.

Cluster 5 contained genes involved in sucrose metabolism, including several sucrose synthase isoforms (Figure 5, Supplementary Table S6). It also harbored genes involved in the regulation of isoprenoid metabolism. Isoprenoids are a large and diverse group of molecules that include photosynthetic pigments (chlorophylls and carotenoids), components of different electron transport chains (plastoquinone, ubiquinone), hormones (abscisic acid, gibberellins, cytokinins and brassinosteroids), membrane-associated phytosterols and antimicrobials (phytoalexins).

Cluster 6 was enriched in pathways related to abiotic stress responses, including various heat-responsive transcription factors and heat-shock proteins (Figure 5, Supplementary Table S6). Most members of this category function as chaperones involved in protein folding, assembly and translocation.

Clusters 7 and 8 exhibit a remarkable over-representation of genes associated to photosynthesis, including components of the light-harvesting complexes, the photosynthetic electron transport chain (PETC) and the Calvin-Benson cycle (Figure 5, Supplementary Table S6). In addition, cluster 7 (528 genes) includes transcripts encoding glycolytic enzymes (e.g., phosphoglycerate kinase) and enzymes involved in homoserine and $\gamma$-aminobutyrate (GABA) synthesis. Cluster 8 (263 genes), in turn, contained transcripts related to cell wall metabolism, although most of the genes belonging to this functional category were grouped in cluster 2 .

A small number of genes showed drought responsiveness only inpfld 252 plants (Figure 6a, Supplementary Table S7). Cluster 9 contained 111 induced genes, with over-representation of transcripts associated to isoprenoid metabolism, whereas cluster 10 included 117 drought-repressed genes, and was enriched in transcripts related to the auxin and minor carbohydrate categories, particularly raffinose synthesis (Figure 6a, Supplementary Table S7).

\subsection{Fld-induced genes that were repressed by water limitation}

Genes grouped in clusters 11-13 (Figure 6b) were induced under control conditions in Stpfld 252 plants, accompanied by drought-related repression in the transformant (cluster 11), or in both lines (clusters 12 and 13). Cluster 11 (240 genes) included genes representing biotic stress and PR proteins, amino acid synthesis and protein and nucleotide metabolism categories (Figure 6b, Supplementary Table S8). Cluster 12 was enriched in photosynthetic genes, particularly components of PSII and its light-harvesting antenna, and cell wall proteins. Finally, cluster 13 included fasciclin-like arabinogalactan proteins, which were reported to participate in fiber initiation and elongation, and to contribute to the integrity of the primary cell wall matrix (Huanget al. 2013). It is worth noting that DE transcripts belonging to the cell wall functional category were also shared with clusters 2 and 8 .

\subsection{Drought-responsive genes primed by Fld expression}

Clusters 14-17 included DE transcripts that responded to water limitation but were also modulated by Fld in the same direction under normal growth conditions (Figure 6c, Supplementary Table S9). Cluster 14 contained 76 transcripts that were induced by chloroplast Fld to the levels attained in drought-stressed WT plants, while genes grouped in cluster 15 (35 members) were further up-regulated by water limitation in both WT and Stpfld 252 plants. DE genes over-represented in these clusters belonged to the ethylene and minor carbohydrate functional categories, including a myo-inositol-1-phosphate synthase (MIPS) that catalyzes the first step of myo-inositol biosynthesis. 
Cluster 16 comprised 127 genes whose expression was repressed by Fld under growth chamber conditions to the levels attained by the drought treatment in both lines (Figure 6c, Supplementary Table S9). Most conspicuous among them was the ethylene responsive factor 5 (ERF5). Finally, cluster 17 included 52 transcripts that were repressed by chloroplast-targeted Fld in the absence of stress, and further by drought in both lines (Figure 6c, Supplementary Table S9), with no specifically enriched functional category.

\subsection{Comparative expression of selected DE transcripts in WT andStpfld252 leaves}

The expression of several DE genes was evaluated by qRT-PCR to validate the results obtained with the RNA microarray experiments. Genes were selected on the basis of their stress responses. Out of $10 \mathrm{DE}$ genes evaluated, 4 showed a strict correlation between the microarray and qRT-PCR data, including the FC, whereas other 5 displayed similar expression patterns in both procedures, but quantitative differences in FC were observed between them (Figure 7). In all 5 cases, the observed differences did not modify cluster assignment.

Chloroplast-targeted Fld prevented the drought-dependent repression of photosynthetic genes to various extents (Figures 5,6). The PSII core complex protein psbY and the photosynthetic Fd isoform 1 were down-regulated in WT leaves under water limitation, as members of cluster 7 (Figure 5). While expression patterns of the two genes determined by microarray analysis and qRT-PCR were similar, the latter procedure gave significantly lower FC values for drought-dependent repression in the wild type (Figure 7). Instead, the two methods yielded a good quantitative correlation for the PSI component psaK belonging to cluster 12 (Figure 6b). Cluster 7 also contained genes encoding two stress-responsive enzymes of central metabolism regarded as drought molecular markers: a granule-bound starch synthase and nitrate reductase 3 (AzedoSilva, Osório, Fonseca \& Correia 2004). Microarray results for both genes were quantitatively validated by qRT-PCR (Figure 7).

Genes primed by Fld expression included the drought-associated transcription factor ATHB7 (cluster 15, Figure 6c), which has been reported to promote stomatal closure among other abiotic stress responses (Ré, Capella, Bonaventure \& Chan 2014). The ATHB7 gene was induced by Fld in the absence of stress and by drought in both lines, displaying one of the highest FC values (Figure 7). The opposite behavior (e.g. , Fldand drought-dependent repression) was exhibited by a gene encoding an aminocyclopropane-1-carboxylate (ACC) oxidase involved in ethylene biosynthesis, which was a member of cluster 17 (Figure 6c). In both cases, microarray analysis and qRT-PCR displayed quantitative differences that did not modify cluster assignment. Another gene displaying a large FC in the transcriptional profiling was $G A S T 1$, encoding a protein involved in plant development and used as a molecular marker to monitor changes in endogenous active gibberellin levels (Shi \& Olszewski 1998). Stress-dependent repression in both WT and Stpfld leaves matches the patterns observed in cluster 2 (Supplementary Figure S5), although lower FC values were obtained for the drought condition in the qRT-PCR experiment compared to the microarray (Figure 7).

Further, two genes encoding components of the proteasomal system (cluster 3, Supplementary Figure S6) were evaluated: subunit PS $\alpha 2 \beta$, which contributes to the assembly of the $20 \mathrm{~S}$ proteasome core complex, and RPN9b, a regulatory subunit of the $26 \mathrm{~S}$ proteasome that is involved in the ATP-dependent degradation of ubiquitinated proteins (Geng, Wenzel \& Tansey 2012). Whilst the RPN9b-encoding transcripts showed a good quantitative correlation between the microarray and qRT-PCR assays, those corresponding to PS $\alpha 2 \beta$ would instead be allocated to cluster 34 instead of cluster 3 on the basis of the qRT-PCR assay (Figure 7). Noteworthy, the change in cluster assignment was caused by a single significant difference in the FC obtained for stressed WT plants (Figure 7).

3.11 Fld presence ameliorated the increase of soluble carbohydrates and amino acid levels in leaves of drought-exposed plants

When plants were grown in the absence of stress, starch and the soluble sugars glucose, fructose and sucrose accumulated to higher levels inStpfld 252 leaves compared to WT siblings (Figure 8a). Water limitation, in turn, led to a major decline of starch in both WT andStpfld 252 leaves, with concomitant increases in soluble sugars (Figure 8a). Unlike carbohydrates, most amino acids accumulated to similar levels in leaves 
from the two genotypes grown under normal conditions, with the conspicuous exception of Pro and its related metabolite pyrroline-5-carboxylate (P5C) whose levels were significantly higher in the transformant (Supplementary Figure S7). Glu and Asp also showed moderately increased contents in Stpfld 252 leaves (Supplementary Figure S7).

With few exceptions (Met, Ala, Glu, GABA and ACC), amino acid contents were up-regulated by water deprivation in WT leaves. These drought-dependent increases were completely prevented by chloroplast Fld for 11 proteinogenic amino acids and ameliorated in three more (Pro, P5C and Thr), whereas Ser and Gly build-up was not affected by the flavoprotein (Figure 8b; Supplementary Figure S7). As expected, drought caused a major increase in the stress marker proline and its precursor P5C, which can act as compatible osmolytes and antioxidants (Szabados \& Savouré 2010). The lower levels observed in Stpfld 252 leaves (Figure 8b; Supplementary Figure S7) suggest that these plants suffered less stress than their WT counterparts.

\subsection{Chloroplast-located Fld improved growth and tuber yield under chronic water restriction}

Observations described in the preceding sections indicate that expression of a chloroplast-targeted Fld increased tolerance to short-term water limitation and attenuated drought transcriptional and metabolic responses in potato leaves. Thus, tuber yield was determined to evaluate possible Fld effects during a long-term water restriction regime with episodic rehydration applied to tuber-producing plants. Impact of drought on tuberization depends not only on the stress intensity but also upon timing, with the most damaging effects on yield occurring when the stress condition was applied at the stolon and tuber initiation stages (Obidiegwu, Bryan, Jones \& Prashar 2015). Therefore, WT and Stpfld 252 plants were grown in soil at $100 \%$ FiC for 30 days, at the time when stolons were set. Water irrigation was interrupted until soil reached $40 \% \mathrm{FiC}$, rehydrated to $70 \% \mathrm{FiC}$ and this protocol repeated for a total treatment of 90 days, when tuberization was extensive in both WT and Stpfld 252 lines (Supplementary Figure S2; see Materials and Methods). The protocol applied was similar to those employed to compare potato genotypes with different drought susceptibilities (Drapal et al. 2017).

Water restriction affected aerial growth in both lines, but significantly less in the transformant. Compared to control watered conditions, stressed WT plants accumulated only $10 \%$ aerial FW at the end of the $90-$ days treatment, whereas Stpfld 252 siblings reached $\sim 25 \%$ (Figure 9a, b). Declines in water potential were significantly lower in Fld-expressing plants under drought (Figure 9c). Differential stress protection by Fld was also reflected at the level of tuber production. Water limitation reduced tuber yield by more than $80 \%$ in WT plants, but less than 70\% in their Stpfld 252 counterparts (Figure 9d, e), resulting in absolute yields of $65 \pm 6$ vs $48 \pm 2 \mathrm{~g}$ per plant for Stpfld 252 and WT lines, respectively (Figure 9e).

\section{DISCUSSION}

Drought is one of the major abiotic stresses affecting agronomic productivity worldwide. Water restriction exerts its negative effect at various physiological and metabolic levels, and the plant protective responses exhibit a comparable complexity. Stomatal closure is among the earliest, aimed at preventing water loss through transpiration (Sperryet al. 2017). An unwanted consequence of this defensive mechanism is the inhibition of gas exchange and $\mathrm{CO}_{2}$ assimilation, which in turn leads to NADPH build-up and blockade of photosynthetic electron transport due to limitation of electron acceptors (oxidized $\mathrm{Fd}$ and $\mathrm{NADP}^{+}$). The excess of reducing equivalents accumulated in the PETC might increase adventitious $\mathrm{O}_{2}$ reduction and ROS propagation, triggering redox-based signaling pathways and eventually oxidative damage (Gómez et al. 2019). Plant responses to this particular aspect of the drought syndrome include a suite of alternative electron transport pathways that dissipate the surplus of excitation energy from the PETC and/or export reducing equivalents to other cellular compartments. They comprise cyclic electron transport, photorespiration, chlororespiration, flavindiiron proteins, the malate valve and the Mehler-Asada cycle (reviewed in Gómezet al. 2019). Scavenging enzymes and metabolites complement the activity of the dissipative systems, limiting ROS accumulation and toxicity. However, drought also causes down-regulation of many photosynthetic components including Fd (Evers et al. 2010; Kondrák et al. 2012; Iovieno et al. 2016). While this response effectively alleviates the hazardous combination of light absorption and highly reduced redox intermediates in a context of elevated 
oxygen levels, the final outcome is further inactivation of photosynthesis.

In many phototrophic microorganisms, environmental stresses such as salinity, heat and iron starvation cause Fd repression (as in plants), accompanied by induction of the equivalent electron shuttle Fld (Pierella Karlusich et al. 2014). This functional substitution allows algae and cyanobacteria to survive and reproduce in hostile environments (Pierella Karlusich et al. 2015). Introduction of Fld in plants via genetic engineering increased tolerance to multiple stresses including drought (Tognetti et al. 2006; Li et al. 2017), complemented Fd-deficient plants (Blanco et al. 2011) and affected the expression of hundreds of genes even at physiological growth conditions and Fd levels (Pierella Karlusich et al. 2017). Moreover, leaves from Fld-expressing tobacco plants displayed a high-light acclimation phenotype (Gómez et al. 2020). These promising results encouraged application of the Fld approach to crops and to the most agronomically relevant stress, drought.

In the present study, we demonstrate that potato plants expressing a plastid-targeted Fld were more tolerant to a short-term drought regime, as reflected by significant preservation of photosynthetic activity (Figure 1) and lower ROS build-up in plastids of Stpfld plants (Figure 2). Drought stress activates a wide array of responses in plants, which together with constitutive traits determine whether the plant will be able to cope with the adverse situation (André et al. 2009). To gain further knowledge on the mechanism of tolerance conferred by Fld, a transcriptomic analysis was carried out on watered and drought-stressed WT and Stpfld 252 potato plants. Since chloroplast Fld could prevent early effects of drought treatment (Figures 1, 2), we chose a 3-day water restriction regime to monitor initial stress responses.

Expression of the plastid-targeted flavoprotein, per se, resulted in significant changes in the expression levels of 1675 genes under control conditions, representing pathways that presumably respond to chloroplastdependent redox-based regulation (Figure 3; Supplementary Table S2). A similar observation has been previously reported for Fld-expressing tobacco plants in terms of the fraction of DE transcripts (5-6\% of total leaf-expressed genes) and, as revealed by functional enrichment analysis, in the pathways over-represented among them (Pierella Karlusich et al. 2017).

Protein degradation via the proteasome is the most conspicuous enriched category. A total of 67 proteasomal genes were induced in Stpfldplants, 48 of which showed a similar behavior in tobacco (Supplementary Figure S8; Pierella Karlusich et al. 2017), suggesting that up-regulation of this pathway is a general plant response to changes in chloroplast redox poise. Proteasome activity has been shown to modulate several key plant responses to both developmental and environmental stimuli (Stone 2014; Bahmani, Kim, Lee \& Hwang 2017; Kovács et al. 2017), indicating that this particular effect of Fld might have profound consequences for plant growth and welfare. Research is currently underway to evaluate these possibilities. In tobacco, most of the proteasomal genes induced by Fld were up-regulated by biotic stress in the wild type (Pierella Karlusich et al. 2017), suggesting that Fld presence primed the plant against subsequent pathogen challenge. Instead, water limitation had little or no effect on proteasomal expression in WT potato (Supplementary Figure S6). Then, induction of this pathway was not involved in the differential drought tolerance displayed by Stpfld lines.

Fld-dependent repression of genes involved in ethylene metabolism was also common to both species, including transcription factors of the apetala2/ERF family, 13 in potato (Figure 3; Supplementary Table S2) and 11 in tobacco (Pierella Karlusich et al. 2017). Common transcriptional responses to Fld presence were observed for 10 of these regulatory factors in the two species, including members of the ERF1, ERF4 and ERF5 sub-families (Supplementary Table S2).

After 3 days of water deprivation, 5701 transcripts were differentially expressed in WT plants, but only 4097 in Fld-expressing siblings (Figure 4). Then, the overall effect of Fld presence was to mitigate the changes in gene expression driven by the drought treatment; either induction or repression, suggesting that plants expressing chloroplast Fld suffered less stress than their WT counterparts, and accordingly displayed an attenuated response that affected many functional categories, as illustrated in Supplementary Figure S9. It is likely that protection of photosynthesis and other metabolic routes contributes to this regulatory effect, presumably by limiting ROS build-up (Figure 2) that might signal subsequent stress responses (Foyer, Ruban 
\& Noctor 2017; Gómez et al. 2019). There is no strict correlation between ROS levels and the magnitude of gene expression reprogramming, but it is expected that decreasing ROS propagation will result in a weaker response as observed in our microarray assay.

Down-regulation of genes encoding photosynthetic components is an universal feature of water limitation (Evers et al. 2010; Kondráket al. 2012; Zhang et al. 2014; Iovieno et al.2016), which aggravates the direct inhibition of photosynthesis through stomatal closure and acceptor side limitation. Under our conditions, 3 days of treatment were sufficient to cause significant repression of transcripts coding for members of the light-harvesting complexes, the PETC and the Calvin-Benson cycle (Figures 4-6), even though decrease of photosynthetic activity was only moderate at this stage (Figure 1b), presumably reflecting slow turnover of the corresponding proteins. Fld expression provided partial or complete protection against repression. It is worth noting, within this context, that photosynthesis was over-represented as a functional category in clusters 7, 8 and 12 (Figures 5,6), but not in cluster 2, which harbored those genes whose drought-dependent repression was not affected by Fld presence (Supplementary Figure S5), underscoring the relevance of the flavoprotein for the preservation of photosynthetic activity in the stressed plants.

A most conspicuous functional category among induced genes was, not unexpectedly, stress, enriched in clusters 1, 5, 6 and 11 (Figures 5, 6; Supplementary Figure S5), indicating that Fld mitigated the stress response of the plant in most cases. While abiotic stress concentrated specifically in clusters 1 and 6 , biotic stress was over-represented in clusters 5 and 11. They included transcripts encoding PR proteins, which have been defined as gene products induced during pathogen infection or wounding. Members of the PR-6, PR-2 (endo- $\beta$-1,3-glucanases) and PR-12 (defensins) families were present in cluster 11, and chitinases in cluster 5 .

Genes found in clusters 14-17 responded to Fld presence under control conditions in the same direction as they did in the wild type under drought (Figure 6c). We coined the term priming to describe genetic traits displaying this behavior (Pierella Karlusich et al. 2017). While mitigation of drought responses by Fld might be a consequence of diminished stress sensed by the plant due to productive interaction of the flavoprotein with photosynthesis and other metabolic routes, priming might actually help the plant to better cope with the environmental challenge, thus representing a cause of the increased drought tolerance. Indeed, priming has been reported to substantially contribute to the phenotypes of drought-tolerant barley varieties, which exhibited stressed-like expression patterns in the absence of stress (Janiaket al. 2018). As in our case, drought caused stronger transcriptional changes in sensitive barley genotypes compared to tolerant varieties (Janiak et al. 2018; You et al. 2019; Zenda et al. 2019). Enriched functional categories among primed potato genes included induction of myo-inositol synthesis (Figure 6c). Overexpression of a MIPS-encoding gene enhanced inositol levels and salt stress tolerance in Arabidopsis, tobacco and rice (Tan, Wang, Xiang, Han \& Guo 2013; Joshi, Ramanarao \& Baisakh 2013; Kusuda et al.2015).

The effects of drought on cell wall metabolism and architecture are complex and depend on the plant species, genotype and age (Le Gallet al. 2015). Figure 4 shows that under the conditions employed, the overall effect of water limitation was repressive, with only marginal protection by Fld. Cluster analysis, in turn, confirmed that most functional categories related to cell wall metabolism grouped in cluster 2 (Supplementary Figure S5), although they were also found in clusters 8, 12 and 13 (Figures 5, 6). In all cases, inclusion in these clusters implied that the relevant genes were down-regulated by drought in the WT, the transformant or both. Genes encoding pectin methylesterases, xyloglucan endotransglucosylases/hydrolases and expansins were extensively represented in these categories (Supplementary Tables S4, S6, S7), indicating that both matrix properties and hemicellulose deposition were compromised by the drought treatment (Tenhaken 2015; Le Gall et al. 2015). The results also suggest that the stress tolerance conferred by Fld expression was not related to protection of cell wall metabolism.

Transcriptional profiles of plants exposed to drought stress have been reported for various crop species including barley, maize, cotton, rice, tomato and potato (Zhang et al. 2014; Iovieno et al. 2016; Janiak et al. 2018; Hasan et al. 2019; Zenda et al.2019; Yang et al. 2019). In those studies, RNA was usually collected at stages of the treatments in which plants already exhibited stress symptoms. Comparison of the functional 
categories over-represented among DE genes in drought-stressed potatoes with those found in our analysis revealed many similarities such as repression of photosynthetic genes and transcripts associated to signaling by receptor kinases (Evers et al. 2010; Kondrák et al. 2012). In addition, stress-repressed genes involved in cell wall metabolism in our transcriptional profiling were the same as those reported by Evers et al. (2010), and some genes involved in abiotic stress such as heat-shock proteins were also shared in several studies (Evers et al. 2010; Zhang et al. 2014). The results suggest that modulation of these stress-responsive genes was initiated early and maintained as the adverse condition proceeds. Direct comparison of early vs. late drought responses in Arabidopsis further supports this contention (Harb, Krishnan, Ambavaram \& Pereira 2010).

Phenotypic effects exerted by chloroplast-located Fld were also evident in the levels of leaf carbohydrates and amino acids. Increased accumulation of transient starch in the absence of stress might result from the higher photosynthetic activity displayed by pfld leaves (Tognetti et al. 2006; Ceccoli et al. 2012; Rossi et al. 2017); its preservation under drought from a lower repression of photosynthetic genes (Figures 5, 6), including starch synthase (Supplementary Table S6; Figure 7). Water limitation led to a strong increase in amino acid levels, most conspicuously Pro, in WT leaves, an effect that was significantly attenuated by Fld presence (Figure 8b). Amino acids synthesized from oxaloacetate as Asn, Asp, Lys, Ile and Thr were induced in WT leaves under drought. A similar response was observed in the 2-oxoglutarate-driven pathway, with increased levels of Pro and Gln only in the WT and Glu in both genotypes under water deprivation. Up-regulation of nitrogen mobilization has been associated with drought and other abiotic stresses (Zhang, Meng, Li \& Zhao 2018), with Gln/Glu and Asn/Asp ratios being customarily used as markers of nitrogen cycling (Goel \& Singh 2015). In our case, they increased significantly in WT leaves under drought (4.3and 1.9-fold, respectively); but remained nearly the same in Stpfld 252 plants (Supplementary Figure S10). The results suggest that nitrogen mobilization was involved in this short-term water deficit condition, in agreement with the up-regulation of genes involved in nitrogen uptake and assimilation observed in similar drought assays (Evers et al. 2010; Goel \& Singh 2015).

Finally, improvement of physiological and molecular stress responses by Fld presence in chloroplasts resulted in increased tuber yield under a long-term non-lethal water restriction protocol (Figure 9). While most drought- and Fld-dependent effects occurred and were monitored in leaves, it is likely that preservation of photosynthesis and other central metabolic pathways $(e . g .$, glycolysis and starch synthesis) under water limitation favored production and transport of photosynthates from source to sink tissues. Further research will be necessary to identify the mechanisms by which the better biochemical performance of Stpfld leaves translated into improved tuber yield, and to evaluate the quality of the resulting tubers in terms of nutritional value and organoleptic properties.

Taken together, our results indicate that the Fld technology constitutes a remarkable tool to improve potato production under less-than-optimal conditions. Field trials are required to properly evaluate this possibility and its agronomic relevance.

\section{LEGENDS TO FIGURES}

Figure 1. Phenotypes and photosynthetic activities of WT and Fld-expressing potato plants under short-term drought treatment. Thirty-days old plants were exposed to hydric stress under growth chamber conditions by interrupting irrigation. (a) Plants were photographed after 14 days of water withdrawal. (b-d) Photosynthetic parameters $F^{\prime}{ }_{\mathrm{v}} / F^{\prime}{ }_{\mathrm{m}}$ (b), PSII (c) and NPQt (d) were determined at the indicated days of treatment, as described in Materials and Methods. Values are means \pm SE of 4-6 biological replicates, and asterisks indicate statistically significant differences with the wild type using ANOVA and Tukey's Multiple Comparison Test at $P$ [?] $0.1\left(^{*}\right)$ or $P$ [?] $0.05\left(^{* *}\right)$.

Figure 2. ROS accumulation in WT and Fld-expressing potato leaves under short-term drought treatment. ROS were visualized by fluorescence microscopy after leaf infiltration with DCFDA. (a) Confocal microscopy analysis of subcellular ROS accumulation in leaves of 30-days old WT and Stpfld252 plants after 0, 3 and 14 days of water withdrawal. Images show ROS fluorescence (left, green), chlorophyll auto-fluorescence 
(middle, magenta), and the merge of the two channels (right). Scale bar: $40 \mu \mathrm{m}$. Quantification of ROS levels in whole-leaf tissue (b) and chloroplasts (c) of WT and Stpfld252plants. Results are means \pm SE of 5 replicates, and asterisks indicate statistically significant differences between lines using ANOVA and Tukey's Multiple Comparison Test ( $P$ [?] 0.05).

Figure 3. Effect of Fld expression on the potato transcriptome.Upper part: pie charts showing the number of genes that were induced $(\mathrm{FC}>2$ and FDR $<0.05)$ or repressed $(\mathrm{FC}<0.5$ and FDR $<$ $0.05)$ by Fld in leaves of 30-days oldStpfld252 plants under growth chamber conditions. Lower part: overrepresentation analysis of Mapman functional among Fld-responsive genes. The analysis was carried out separately for induced and repressed genes (Fisher's exact test with Bonferroni correction and FDR $<0.05$ ). The list of genes and their corresponding descriptions, functional assignments and FC values are described in Supplementary Table S2.

Figure 4. Drought stress led to extensive transcriptional reprogramming in both WT and Fld-expressing plants.Venn diagrams of genes differentially expressed (DE) in response to drought are shown in the right upper corner. Genes were defined as induced when FC $>2$ and FDR $<0.05$, and as repressed when $\mathrm{FC}<0.5$ and $\mathrm{FDR}<0.05$. On the left, list of functional categories with over-represented DE genes during the response of WT and Stpfld252 plants to drought. The analysis was carried out separately for induced and repressed genes (Fisher's exact test with Bonferroni correction and FDR $<0.05$ ). The list of genes and their corresponding descriptions, assignments and FC values are described in Supplementary Table S3.

Figure 5. Clusters containing genes in which the drought response was abolished or ameliorated by Fld expression. Each gray line of the charts corresponds to a particular gene, and the dark line represents the average behavior of all the genes contained in each cluster. Labelings in the abscissa correspond to the WT line under control (WT.C) and drought conditions (WT.D), and the Stpfld252line under control (Fld.C) and drought conditions (Fld.D); those in the ordinates correspond to the FC values in $\log _{2}$ scale. For each pairwise comparison between lines or treatments, genes were defined as induced when $\mathrm{FC}>2$ and FDR $<0.05$, and repressed when FC $<0.5$ and FDR $<0.05$. The total number of genes in each cluster is indicated above the corresponding panel, and theover-represented functional categories are shown below (analyzed using Fisher's exact test with Bonferroni correction and FDR $<0.05$ ). The list of genes belonging to these clusters and their corresponding descriptions, functional assignments and FC values in the multiple comparison tests are described in Supplementary Table S6.

Figure 6. Clusters containing DE genes with various interactions between treatment and Fld. (a) Genes affected by drought exclusively in Fld-expressing plants. (b) Genes regulated by Fld and drought in opposite directions. (c) Drought-responsive genes which were already primed by Fld in the absence of stress. Each gray line of the charts corresponds to a particular gene, and the dark line represents the average behavior of all cluster genes. Labelings of the abscissa and ordinates, total number of genes in each cluster and list of over-represented pathways are indicated as in Figure 5. Further details are provided in Supplementary Tables S7 (a), S8 (b) and S9 (c).

Figure 7. Comparison of expression patterns of selected DE genes as determined by microarray analysis (dark circles) and qRT-PCR (open circles). Labelings in the abscissa correspond to the WT line under control (WT.C) and drought conditions (WT.D), and theStpfld 252 line under control (Fld.C) and drought conditions (Fld.D); those in the ordinates correspond to the $\mathrm{FC}$ values in $\log _{2}$ scale relative to those of WT siblings under control conditions (WT.C). Each data point of qRT-PCR determinations represents the mean and standard deviation of 4-5 biological replicates. Within each line, a statistically significant effect relative to WT.C is indicated as a gray asterisk for microarray data (FDR $<0.05$ and FC $>2$ or FC $<0.5)$ and a black asterisk for qPCR data $(P$ values $<0.05$ according to ANOVA test). psaK, photosystem I reaction center subunit; Fd1, Fd isoform 1; psbY, photosystem II core complex protein; GBSSI, granulebound starch synthase; NR3, nitrate reductase 3; ATHB7, Arabidopsis homeobox-leucine zipper protein HB7; ACO1, aminocyclopropane-1-carboxylate oxidase 1; GAST1, gibberellin-regulated protein GAST1; PSA2B, proteasome subunit PS $\alpha 2 \beta \cdot R P N 9 b, 26 S$ proteasome subunit RPN9b. 
Figure 8. Fld presence attenuates build-up of soluble sugars and amino acids under water limitation. Extracts were prepared from leaves of 30-days old plants at 3 days of water deprivation and from their watered controls ( $8 \mathrm{~h}$ within the light period), and the levels of the indicated sugars and amino acids were determined as described in Materials and Methods. (a) Carbohydrate contents are given as means $\pm \mathrm{SE}$ of 5-8 independent plants. Statistically significant differences between lines are shown by asterisks and were determined using ANOVA and Tukey's Multiple Comparison Test $(P<0.05)$. FW, fresh weight. (b) Heat map of amino acids assayed for the different lines and treatments. Color scale corresponds to the standardized scores (dark blue for low values, yellow for high values). Quantitative data of amino acid determinations are shown in Supplementary Figure S7. C, control conditions; D, drought. Heat maps were produced in $\mathrm{R}$ language using the heatmap. 2 function of the gplots package.

Figure 9. Phenotypes of WT and Fld-expressing potato plants under long-term drought treatment. Plants were grown in soil for 30 days at $100 \%$ FiC. Water irrigation was then interrupted until soil reached $40 \% \mathrm{FiC}$, rehydrated to $70 \% \mathrm{FiC}$ and this protocol repeated for a total treatment of 90 days (Supplementary Figure S2). (a) Plants were photographed after 90 days of drought treatment. (b) Fresh weight of aerial parts. (c) Water potential. (d) Representative photograph of tubers. (e) Tuber yield. Results are means \pm SE of 5 replicate plants, and asterisks indicate statistically significant differences between lines using non-parametric Mann-Whitney test (P [?] 0.1).

\section{SUPPLEMENTARY MATERIAL}

Supplementary Figure S1. Fld expression in leaves of transgenic potato plants. (a) Schematic diagram of the T-DNA region of the vector used for transformation. The fld gene was translationally fused to the transit peptide (TP) of pea FNR for plastid targeting, and placed between the constitutive CaMV $35 \mathrm{~S}$ promoter and the transcriptional terminator of the nopaline synthase gene (NOS terminator) in the binary vector pCAMBIA 2200 (Tognetti et al., 2006). (b) Fld expression in leaf tissue from independent Stpfld lines as determined by SDS-PAGE and immunoblot using Fld antisera (see Materials and Methods). The fourth fully expanded leaves of 30-days old plants were used to prepare the extracts. Soluble fractions corresponding to $10 \mathrm{mg} \mathrm{FW}$ were loaded in each lane except for the control, which corresponds to 0.8 pmol of purified Fld (arrow). The intensities of the bands co-migrating with mature-sized Fld were quantified with the Image J software, and expressed in arbitrary units (AU). Data shown are means +- SE of three independent experiments.

Supplementary Figure S2. Long-term drought protocol.Plants were grown in 15-L soil pots and irrigation was stopped at 30 days post germination to initiate the drought regime. One hundred per cent FC was defined as the amount of water necessary to completely moisten a 15-L dry soil pot, and we determined FC throughout the experiment by subtracting weights at each time point of the treatment from the initial weight of each pot.

Supplementary Figure S3. Relative water content during short-term drought. Thirty-days old plants were subjected to water stress and RWC was measured at 0,3 and 14 days post drought, as described in Materials and Methods. Data are means +- SE of 3 biological replicates. Statistically significant differences with the wild type are shown by asterisks and were determined using ANOVA and Tukey's Multiple Comparison Test $(P<0.1)$.

Supplementary Figure S4. Low-populated clusters not shown in the main text Figures. Each gray line of the charts corresponds to a particular gene, and the dark line represents the average behavior of all cluster genes. Labelings of the abscissa and ordinates, total number of genes and the list of pathways overrepresented in each cluster are indicated as in the legend to Figure 5. Each cluster is displayedvis-a-vis to other that exhibits the contrasting expression behavior (e.g ., induction vs. repression), with the exceptions of clusters 44, 45 and 46, whose opposite clusters corresponds to 11, 12 and 13 (Fig. 6b), respectively.

Supplementary Figure S5. Clusters formed by genes induced or repressed by drought independently of genotype. Each gray line corresponds to a particular gene, and the dark lines represent the average behavior of all the genes contained in each cluster. Labelings of the abscissa and ordinates, total 
number of genes and pathways over-represented in each cluster are indicated as in the legend to Figure 5. The list of genes belonging to these clusters and their corresponding descriptions, assignments and FC values in the multiple comparison tests are described in Supplementary Table S4.

Supplementary Figure S6. Clusters formed by Fld-modulated genes not responsive to the drought treatment. Each gray line of the charts corresponds to a particular gene, and the dark line represents the average behavior of all cluster genes. Labelings of the abscissa and ordinates, total number of genes and list of pathways over-represented in each cluster are indicated as in Figure 5. The corresponding data are given in Supplementary Table S5.

Supplementary Figure S7. Amino acid profiling in leaves from WT and Fld-expressing potato plants. Amino acid contents were determined in leaves of 30-days old plants as described in Materials and Methods. Levels are reported as means +- SE of 5-8 plants. Statistically significant differences between lines are shown by asterisks and were determined using ANOVA and Tukey's Multiple Comparison Test $(P<$ $0.05)$.

Supplementary Figure S8. Comparison of DE genes associated to the proteasome in leaves from Fld-expressing potato and tobacco plants grown under normal conditions. Mapman representations of the plant proteolytic pathway associated to the proteasome are shown for potato (a) and tobacco (b). Each colored square represents a gene that is induced (red; FC $>2$ and FDR $<0.05$ ) or repressed (green; FC $<0.5$ and FDR $<0.05$ ) by Fld expression under control conditions. Only DE genes are displayed. The color code is shown in a rectangle using a $\log _{2}$ scale. Genes coding for components of the 26S proteasome are squared. Tobacco data were taken from Pierella Karlusich et al. (2017).

Supplementary Figure S9. Comparison of drought-responsive DE genes in WT and Fldexpressing potato plants. The major functional categories of central and secondary metabolism are shown in Mapman representations. Each colored square represents a gene that is induced (red; FC $>2$ and FDR $<0.05$ ) or repressed (green; FC $<0.5$ and FDR $<0.05$ ) by drought in WT (a) and Fld-expressing (b) leaves. Only DE genes are displayed. Color schemes are shown as in Supplementary Figure S8.

Supplementary Figure S10. Drought modified the ratios of amino acids related to nitrogen mobilization in WT but not Stpfldplants. Ratios of Gln to Glu and Asn to Asp were determined in leaves of 30-days old plants after 3 days of water restriction and their watered controls, as described in Materials and Methods. Levels are reported as means +- SE of 5-8 plants. Statistically significant differences between lines are shown by asterisks and were determined using ANOVA and Tukey's Multiple Comparison Test $(P<0.05)$.

Supplementary Table S1. Primer sets used for qRT-PCR determinations.

Supplementary Table S2. List of potato genes with altered expression patterns in response to Fld (Figure $3)$. Gene ID and description, Mapman pathway annotation and FC (in $\log _{2}$ scale) are shown. The color code indicates statistical significance in induction ( $\mathrm{red}, \log _{2} \mathrm{FC}>1$, FDR $<0.05$ ) or repression (green, $\log _{2} \mathrm{FC}<$ -1$, FDR $<0.05)$. Genes belonging to various pathways are displayed repetitively.

Supplementary Table S3. List of potato genes with altered expression patterns in response to drought in WT and Fld-expressing leaves (Figure 4). Gene ID and description, Mapman pathway annotation and FC (in $\log _{2}$ scale) are shown. The color code indicates statistical significance in induction (red, $\log _{2} \mathrm{FC}>$ $1, \mathrm{FDR}<0.05$ ) or repression (green, $\log _{2} \mathrm{FC}<-1, \mathrm{FDR}<0.05$ ). Genes belonging to various pathways are displayed repetitively.

Supplementary Table S4. List of potato genes belonging to clusters formed by drought-induced or repressed genes independently of genotype (Supplementary Figure S5). Gene ID and description, Mapman pathway annotation, and $\mathrm{FC}$ (in $\log _{2}$ scale) for each of the four line/treatment comparisons are shown. The color code indicates statistical significance in induction (red, $\log _{2} \mathrm{FC}>1$, FDR $<0.05$ ) or repression (green, $\left.\log _{2} \mathrm{FC}<-1, \mathrm{FDR}<0.05\right)$. Genes belonging to various pathways are displayed repetitively. 
Supplementary Table S5. List of potato genes belonging to clusters formed by genes regulated by Fld whose expression was not affected by drought (Supplementary Figure S6). Gene ID and description, Mapman pathway annotation, and FC (in $\log _{2}$ scale) for each of the four line/treatment comparisons are shown. The color code indicates statistical significance in induction (red, $\log _{2} \mathrm{FC}>1$, FDR $<0.05$ ) or repression (green, $\left.\log _{2} \mathrm{FC}<-1, \mathrm{FDR}<0.05\right)$. Genes belonging to various pathways are displayed repetitively.

Supplementary Table S6. List of potato genes belonging to clusters formed by drought responses abolished or ameliorated in the presence of chloroplast Fld (Figure 5). Gene ID and description, Mapman pathway annotation, and FC (in $\log _{2}$ scale) for each of the four line/treatment comparisons are shown. The color code indicates statistical significance in induction ( $\mathrm{red}, \log _{2} \mathrm{FC}>1, \mathrm{FDR}<0.05$ ) or repression (green, $\left.\log _{2} \mathrm{FC}<-1, \mathrm{FDR}<0.05\right)$. Genes belonging to various pathways are displayed repetitively.

Supplementary Table S7. List of DE potato genes that responded to drought only in the presence of chloroplast Fld (Figure 6a). Gene ID and description, Mapman pathway annotation, and FC (in $\log _{2}$ scale) for each of the four line/treatment comparisons are shown. The color code indicates statistical significance in induction (red, $\log _{2} \mathrm{FC}>1$, FDR $<0.05$ ) or repression (green, $\log _{2} \mathrm{FC}<-1$, FDR $<0.05$ ). Genes belonging to various pathways are displayed repetitively.

Supplementary Table S8. List of potato genes regulated by drought and Fld in opposite directions (Figure 6b). Gene ID and description, Mapman pathway annotation, and FC (in $\log _{2}$ scale) for each of the four line/treatment comparisons are shown. The color code indicates statistical significance in induction (red, $\log _{2} \mathrm{FC}>1, \mathrm{FDR}<0.05$ ) or repression (green, $\log _{2} \mathrm{FC}<-1$, FDR $<0.05$ ). Genes belonging to various pathways are displayed repetitively.

Supplementary Table S9. List of potato genes belonging to clusters formed by drought-responsive genes which were already primed by Fld in the absence of stress (Figure 6c). Gene ID and description, Mapman pathway annotation, and $\mathrm{FC}$ (in $\log _{2}$ scale) for each of the four line/treatment comparisons are shown. The color code indicates statistical significance in induction (red, $\log _{2} \mathrm{FC}>1$, FDR $<0.05$ ) or repression (green, $\left.\log _{2} \mathrm{FC}<-1, \mathrm{FDR}<0.05\right)$. Genes belonging to various pathways are indicated repetitively.

\section{REFERENCES}

Ahmad R., Kim Y.H., Kim M.D., Kwon S.Y., Cho K., Lee H.S. \& Kwak S.S. (2010) Simultaneous expression of choline oxidase, superoxide dismutase and ascorbate peroxidase in potato plant chloroplasts provides synergistically enhanced protection against various abiotic stresses.Physiologia Plantarum, 138 , 520-533.

Andre C.M., Schafleitner R., Legay S., Lefevre I., Aliaga C.A.A., Nomberto G. \& Evers D. (2009) Gene expression changes related to the production of phenolic compounds in potato tubers grown under drought stress. Phytochemistry, $\mathbf{7 0}, 1107-1116$.

Azedo-Silva J., Osorio J., Fonseca F. \& Correia M.J. (2004) Effects of soil drying and subsequent re-watering on the activity of nitrate reductase in roots and leaves of Helianthus annuus. Functional Plant Biology, $\mathbf{3 1}$ ,611-621.

Bahmani R., Kim D.G., Lee B.D. \& Hwang S. (2017) Over-expression of tobacco UBC1 encoding a ubiquitinconjugating enzyme increases cadmium tolerance by activating the 20S/26S proteasome and by decreasing $\mathrm{Cd}$ accumulation and oxidative stress in tobacco (Nicotiana tabacum). Plant Molecular Biology, 94 , 433-451.

Baker N.R. (2008) Chlorophyll Fluorescence: A Probe of Photosynthesis In Vivo. Annual Review of Plant Biology, 59, 89-113.

Blanco N.E., Ceccoli R.D., Segretin M.E., Poli H.O., Voss I., Melzer M., .. C Carrillo N. (2011) Cyanobacterial flavodoxin complements ferredoxin deficiency in knocked-down transgenic tobacco plants.Plant Journal, 65 , 922-935.

Buchanan, B.B., Gruissem, W. \& Jones R.L. (2015) Biochemistry \& Molecular Biology of Plants., 2nd edition. Rockville, MD: American Society of Plant Physiologists. 
Ceccoli R.D., Blanco N.E., Segretin M.E., Melzer M., Hanke G.T., Scheibe R., .. C Carrillo N. (2012) Flavodoxin displays dose-dependent effects on photosynthesis and stress tolerance when expressed in transgenic tobacco plants. Planta, $236,1447-1458$.

Cheng Y.J., Deng X.P., Kwak S.S., Chen W. \& Eneji A.E. (2013) Enhanced tolerance of transgenic potato plants expressing choline oxidase in chloroplasts against water stress. Botanical Studies,54 .

Cruz De Carvalho M.H. (2008) Drought stress and reactive oxygen species: Production, scavenging and signaling. Plant Signaling and Behavior, $3,156-165$.

Czarnocka W. \& Karpiński S. (2018) Friend or foe? Reactive oxygen species production, scavenging and signaling in plant response to environmental stresses. Free Radical Biology and Medicine,122 , 4-20.

Drapal M., Farfan-Vignolo E.R., Gutierrez O.R., Bonierbale M., Mihovilovich E. \& Fraser P.D. (2017) Identification of metabolites associated with water stress responses in Solanum tuberosum L. clones.Phytochemistry, 135, 24-33.

Edgar R., Domrachev M. \& Lash A.E. (2002) Gene Expression Omnibus: NCBI gene expression and hybridization array data repository. Nucleic Acids Research, 30 , 207-210.

Eltayeb A.E., Yamamoto S., Elsadig M., Habora E., Yin L., Tsujimoto H. \& Tanaka K. (2011) Transgenic potato overexpressing Arabidopsis cytosolic AtDHAR1 showed higher tolerance to herbicide, drought and salt stresses. Breeding Science, 61, 3-10.

Evers D., Lefvre I., Legay S., Lamoureux D., Hausman J.F., Rosales R.O.G., ... Schafleitner R. (2010) Identification of drought-responsive compounds in potato through a combined transcriptomic and targeted metabolite approach. Journal of Experimental Botany,61 , 2327-2343.

FAO (2014) Food and Agricultural Organization of the United Nations Database. Rome.

Foyer C.H., Ruban A. V. \& Noctor G. (2017) Viewing oxidative stress through the lens of oxidative signalling rather than damage.Biochemical Journal, 474, 877-883.

Le Gall H., Philippe F., Domon J.M., Gillet F., Pelloux J. \& Rayon C. (2015) Cell wall metabolism in response to abiotic stress.Plants, $4,112-166$.

Geng F., Wenzel S. \& Tansey W.P. (2012) Ubiquitin and Proteasomes in Transcription. Annual Review of Biochemistry, 81, 177-201.

Ghaffari M., Shahinnia F., Usadel B., Junker B., Schreiber F., Sreenivasulu N. \& Hajirezaei M. (2016) The Metabolic Signature of Biomass Formation in Barley. Plant $\& 3$ cell physiology,57 , 1943-1960.

Goel P. \& Singh A.K. (2015) Abiotic stresses downregulate key genes involved in nitrogen uptake and assimilation in brassica juncea l.PLoS ONE, 10(11) .

Gómez R., Figueroa N., Melzer M., Hajirezaei M.R., Carrillo N., \& Lodeyro A.F. (2020). Photosynthetic characterization of flavodoxin-expressing tobacco plants reveals a high light acclimation-like phenotype. Biochimica et Biophysica Acta - Bioenergetics, 1861(8) .

Gómez R., Vicino P., Carrillo N. \& Lodeyro A.F. (2019) Manipulation of oxidative stress responses as a strategy to generate stress-tolerant crops. From damage to signaling to tolerance. Critical Reviews in Biotechnology, 39 , 693-708.

Harb A., Krishnan A., Ambavaram M.M.R. \& Pereira A. (2010) Molecular and physiological analysis of drought stress in arabidopsis reveals early responses leading to acclimation in plant growth. Plant Physiology, $154,1254-1271$.

Hasan M.M.U., Ma F., Islam F., Sajid M., Prodhan Z.H., Li F., .. Wang X. (2019) Comparative Transcriptomic Analysis of Biological Process and Key Pathway in Three Cotton (Gossypium spp.) Species Under Drought Stress. International journal of molecular sciences, $\mathbf{2 0}(\mathbf{9})$. 
Huang G.Q., Gong S.Y., Xu W.L., Li W., Li P., Zhang C.J., .. L Li X.B. (2013) A fasciclin-like arabinogalactan protein, GhFLA1, is involved in fiber initiation and elongation of cotton. Plant Physiology, 161 , 1278-1290.

Iovieno P., Punzo P., Guida G., Mistretta C., Van Oosten M.J., Nurcato R., .. Grillo S. (2016) Transcriptomic changes drive physiological responses to progressive drought stress and rehydration in tomato. Frontiers in Plant Science, $7,1-14$.

IPCC (2014). Climate Change 2014: Mitigation of Climate Change Contribution of Working Group III to the Fifth Assessment Report of the Intergovernmental Panel on Climate Change, eds Edenhofer O.R., Pichs-Madruga Y., Sokona E., Farahani S., Kadner K., Seyboth A., et al. Cambridge: Cambridge University Press.

Janiak A., Kwasniewski M., Sowa M., Gajek K., Żmuda K., Kościelniak J. \& Szarejko I. (2018) No time to waste: Transcriptome study reveals that drought tolerance in barley may be attributed to stressed-like expression patterns that exist before the occurrence of stress. Frontiers in Plant Science, 8 .

Joshi R., Ramanarao M.V. \& Baisakh N. (2013) Arabidopsis plants constitutively overexpressing a myoinositol 1-phosphate synthase gene (SaINO1) from the halophyte smooth cordgrass exhibits enhanced level of tolerance to salt stress. Plant Physiology and Biochemistry,65, 61-66.

Kloosterman B., De Koeyer D., Griffiths R., Flinn B., Steuernagel B., Scholz U., .. Bachem C.W.B. (2008) Genes driving potato tuber initiation and growth: Identification based on transcriptional changes using the POCI array. Functional and Integrative Genomics,8 , 329-340.

Kondrák M., Marincs F., Antal F., Juhász Z. \& Bánfalvi Z. (2012) Effects of yeast trehalose-6-phosphate synthase 1 on gene expression and carbohydrate contents of potato leaves under drought stress conditions. $B M C$ Plant Biology, 12, 1-12.

Kovács J., Poór P., Kaschani F., Chandrasekar B., Hong T.N., Misas-Villamil J.C., .. van der Hoorn R.A.L. (2017) Proteasome activity profiling uncovers alteration of catalytic $\beta 2$ and $\beta 5$ subunits of the stress-induced proteasome during salinity stress in tomato roots.Frontiers in Plant Science, 8 , 1-13.

Kuhlgert S., Austic G., Zegarac R., Osei-bonsu I., Hoh D., Chilvers M.I., .. Kramer D.M. (2016) MultispeQ Beta : a tool for phenotyping connected to the open PhotosynQ network Subject Areas : Author for correspondence : Royal Society Open Science,3 , 160592.

Kusuda H., Koga W., Kusano M., Oikawa A., Saito K., Hirai M.Y. \& Yoshida K.T. (2015) Ectopic expression of myo-inositol 3-phosphate synthase induces a wide range of metabolic changes and confers salt tolerance in rice. Plant Science, 232, 49-56.

Li Z., Yuan S., Jia H., Gao F., Zhou M., Yuan N., .. Luo H. (2017) Ectopic expression of a cyanobacterial flavodoxin in creeping bentgrass impacts plant development and confers broad abiotic stress tolerance.Plant Biotechnology Journal, 15, 433-446.

Logemann J., Schell J. \& Willmitzer L. (1987) Improved method for the isolation of RNA from plant tissues. Analytical Biochemistry,163, 16-20.

Mayta M.L., Arce R.C., Zurbriggen M.D., Valle E.M., Hajirezaei M.R., Zanor M.I. \& Carrillo N. (2019) Expression of a Chloroplast-Targeted Cyanobacterial Flavodoxin in Tomato Plants Increases Harvest Index by Altering Plant Size and Productivity. Frontiers in Plant Science,10 , 1-13.

Mayta M.L., Lodeyro A.F., Guiamet J.J., Tognetti V.B., Melzer M., Hajirezaei M.R. \& Carrillo N. (2018) Expression of a Plastid-Targeted Flavodoxin Decreases Chloroplast Reactive Oxygen Species Accumulation and Delays Senescence in Aging Tobacco Leaves. Frontiers in Plant Science, 9 , 1-18.

Muñiz García M.N., Cortelezzi J.I., Fumagalli M. \& Capiati D.A. (2018) Expression of the Arabidopsis ABF4 gene in potato increases tuber yield, improves tuber quality and enhances salt and drought tolerance.Plant Molecular Biology, 98, 137-152. 
Obidiegwu J.E., Bryan G.J., Jones H.G. \& Prashar A. (2015) Coping with drought: Stress and adaptive responses in potato and perspectives for improvement. Frontiers in Plant Science, 6 , 1-23.

Pierella Karlusich J.J., Ceccoli R.D., Graña M., Romero H. \& Carrillo N. (2015) Environmental selection pressures related to iron utilization are involved in the loss of the flavodoxin gene from the plant genome.Genome Biology and Evolution, 7 , 750-767.

Pierella Karlusich J.J., Lodeyro A.F. \& Carrillo N. (2014) The long goodbye: The rise and fall of flavodoxin during plant evolution. Journal of Experimental Botany, 65 , 5161-5178.

Pierella Karlusich J.J., Zurbriggen M.D., Shahinnia F., Sonnewald S., Sonnewald U., Hosseini S.A., ... Carrillo N. (2017) Chloroplast Redox Status Modulates Genome-Wide Plant Responses during the Nonhost Interaction of Tobacco with the Hemibiotrophic Bacterium Xanthomonas campestris pv. vesicatoria. Frontiers in Plant Science, , , 1-21.

Ramšak Ž., Baebler Š., Rotter A., Korbar M., Mozetič I., Usadel B. \& Gruden K. (2014) GoMapMan: integration, consolidation and visualization of plant gene annotations within the MapMan ontology. Nucleic Acids Research, 42, D1167-D1175.

Ré D.A., Capella M., Bonaventure G. \& Chan R.L. (2014) Arabidopsis AtHB7 and AtHB12 evolved divergently to fine tune processes associated with growth and responses to water stress. BMC Plant Biology,14, $1-14$.

Ritchie M.E., Phipson B., Wu D., Hu Y., Law C.W., Shi W. \& Smyth G.K. (2015) limma powers differential expression analyses for RNA-sequencing and microarray studies. Nucleic Acids Research, 43(7),e47.

Rocha-Sosa M., Sonnewald U., Frommer W., Stratmann M., Schell' J. \& Willmitzer L. (1989) Both developmental and metabolic signals activate the promoter of a class I patatin gene. EMBO Journal,8(1) , $23-29$.

Romero A.P., Alarcón A., Valbuena R.I. \& Galeano C.H. (2017) Physiological assessment of water stress in potato using spectral information. Frontiers in Plant Science, $\mathbf{8}$.

Rossi F.R., Krapp A.R., Bisaro F., Maiale S.J., Pieckenstain F.L. \& Carrillo N. (2017) Reactive oxygen species generated in chloroplasts contribute to tobacco leaf infection by the necrotrophic fungusBotrytis cinerea. The Plant Journal, $92,761-773$.

Schindelin J., Arganda-Carreras I., Frise E., Kaynig V., Longair M., Pietzsch T., Cardona A. (2012) Fiji: An open-source platform for biological-image analysis. Nature Methods, 9 , 676-682.

Schmittgen T.D. \& Livak K.J. (2008) Analyzing real-time PCR data by the comparative CT method. Nature Protocols, 3 , 1101-1108.

Shi L. \& Olszewski N.E. (1998) Gibberellin and abscisic acid regulate GAST1 expression at the level of transcription. Plant Molecular Biology, 38 , 1053-1060.

Sperry J.S., Venturas M.D., Anderegg W.R.L., Mencuccini M., Mackay D.S., Wang Y. \& Love D.M. (2017) Predicting stomatal responses to the environment from the optimization of photosynthetic gain and hydraulic cost. Plant Cell and Environment, 40, 816-830.

Stone S.L. (2014) The role of ubiquitin and the $26 \mathrm{~S}$ proteasome in plant abiotic stress signaling. Frontiers in Plant Science, 5 , 1-10.

Szabados L. \& Savouré A. (2010) Proline: a multifunctional amino acid.Trends in Plant Science, 15 , 89-97.

Tan J., Wang C., Xiang B., Han R. \& Guo Z. (2013) Hydrogen peroxide and nitric oxide mediated cold- and dehydration-induced myo-inositol phosphate synthase that confers multiple resistances to abiotic stresses. Plant, Cell and Environment, 36 , 288-299.

Tenhaken R. (2015) Cell wall remodeling under abiotic stress.Frontiers in Plant Science, $\mathbf{5}$. 
Thimm O., Bläsing O., Gibon Y., Nagel A., Meyer S., Krüger P., .. Stitt M. (2004) MAPMAN: A user-driven tool to display genomics data sets onto diagrams of metabolic pathways and other biological processes. Plant Journal, 37 , 914-939.

Tognetti V.B., Palatnik J.F., Fillat M.F., Melzer M., Hajirezaei M.R., Valle E.M. \& Carrillo N. (2006) Functional replacement of ferredoxin by a cyanobacterial flavodoxin in tobacco confers broad-range stress tolerance. Plant Cell, 18 , 2035-2050.

Tognetti V.B., Zurbriggen M.D., Morandi E.N., Fillat M.F., Valle E.M., Hajirezaei M.-R. \& Carrillo N. (2007) Enhanced plant tolerance to iron starvation by functional substitution of chloroplast ferredoxin with a bacterial flavodoxin. Proceedings of the National Academy of Sciences, 104, 11495-11500.

Urban L., Aarrouf J. \& Bidel L.P.R. (2017) Assessing the effects of water deficit on photosynthesis using parameters derived from measurements of leaf gas exchange and of chlorophyll a fluorescence. Frontiers in Plant Science, $8,2068$.

Usadel B., Nagel A., Steinhauser D., Gibon Y., Bläsing O.E., Redestig H., .. Stitt M. (2006) PageMan: An interactive ontology tool to generate, display, and annotate overview graphs for profiling experiments. $B M C$ Bioinformatics, $7,535-535$.

Vasquez-Robinet C., Mane S.P., Ulanov A. V, Watkinson J.I., Stromberg V.K., De Koeyer D., ... Grene R. (2008) Physiological and molecular adaptations to drought in Andean potato genotypes. Journal of Experimental Botany, 59 , 2109-2123.

Visser R.G.F., Bachem C.W.B., de Boer J.M., Bryan G.J., Chakrabati S.K., Feingold S., .. Tang X. (2009) Sequencing the Potato genome: Outline and first results to come from the Elucidation of the sequence of the world's third most important food crop. American Journal of Potato Research, 86 , 417-429.

Wickham H. (2009) ggplot2: Elegant Graphics for Data Analysis. New York: Springer.

Yang S., Xu K., Chen S., Li T., Xia H., Chen L., .. Luo L. (2019) A stress-responsive bZIP transcription factor OsbZIP62 improves drought and oxidative tolerance in rice. BMC Plant Biology, 19 , 260.

You J., Zhang Y., Liu A., Li D., Wang X., Dossa K., ... Zhang X. (2019) Transcriptomic and metabolomic profiling of drought-tolerant and susceptible sesame genotypes in response to drought stress. BMC Plant Biology, $19,1-16$.

Zenda T., Liu S., Wang X., Liu G., Jin H., Dong A., .. Duan H. (2019) Key maize drought-responsive genes and pathways revealed by comparative transcriptome and physiological analyses of contrasting inbred lines. International Journal of Molecular Sciences,20 , 1-30.

Zhang C., Meng S., Li M. \& Zhao Z. (2018) Transcriptomic insight into nitrogen uptake and metabolism of Populus simonii in response to drought and low nitrogen stresses. Tree physiology, 38 , 1672-1684.

Zhang N., Liu B., Ma C., Zhang G., Chang J., Si H. \& Wang D. (2014) Transcriptome characterization and sequencing-based identification of drought-responsive genes in potato. Molecular Biology Reports,41, $505-517$.

Zingaretti S.M., Inacio M.C., de Matos Pereira L., Paz T.A. \& de Castro Franca S. (2013) Water Stress and Agriculture. In Responses of Organisms to Water Stress, Sener Akın. InTech.

Zurbriggen M.D., Tognetti V.B., Fillat M.F., Hajirezaei M.-R., Valle E.M. \& Carrillo N. (2008) Combating stress with flavodoxin: a promising route for crop improvement. Trends in biotechnology, 26, 531-7. 


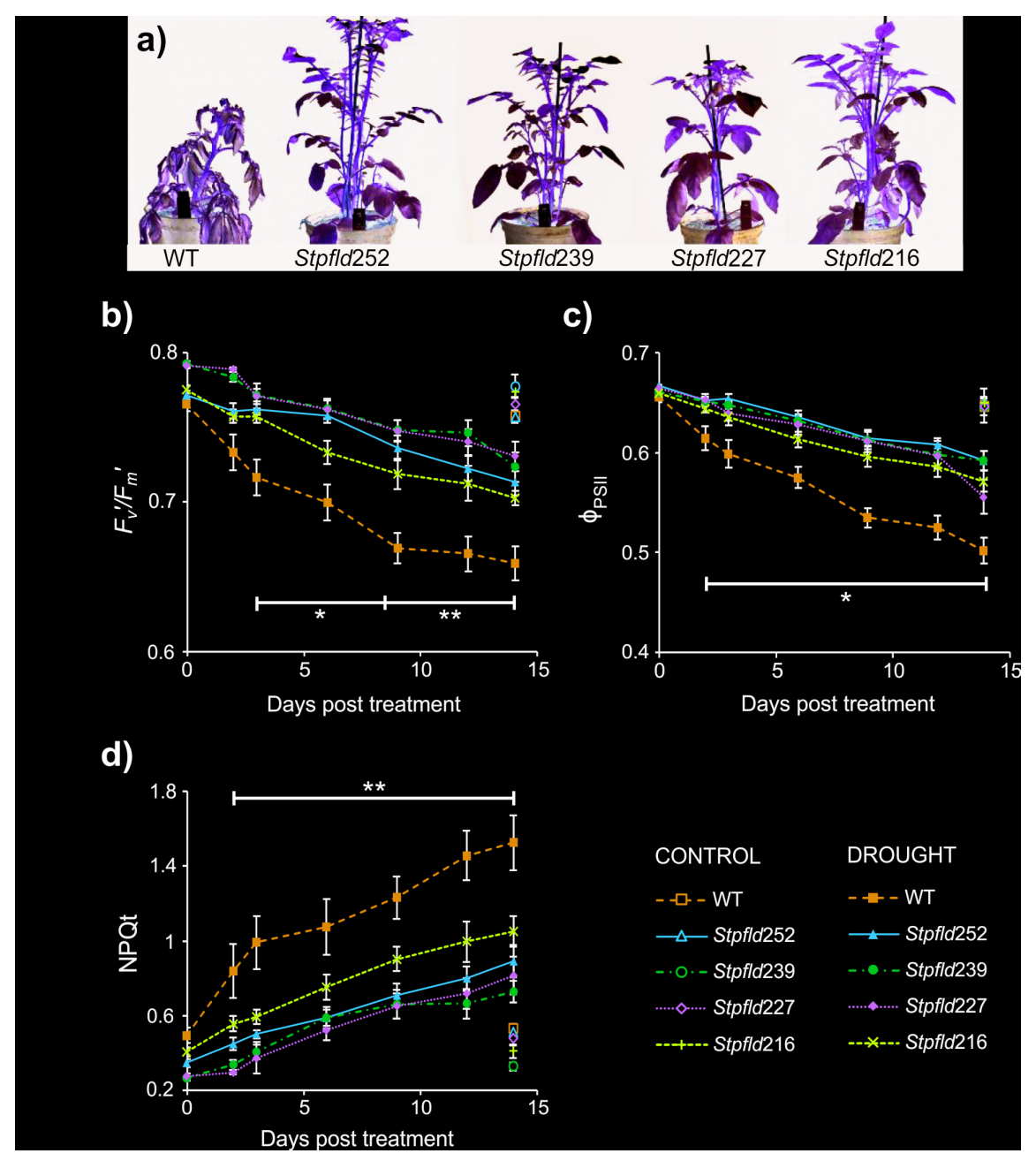




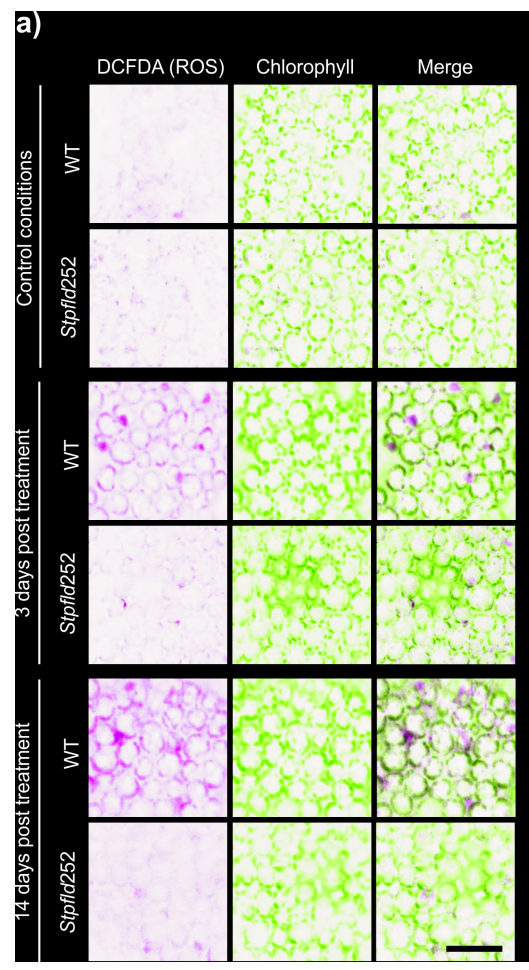

Fld-induced genes

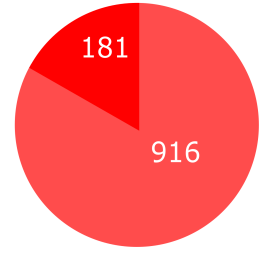

$\square \mathrm{FC}>4 \quad \square$ 4 $>$ FC $>2$

cell wall

cell wall.cell wall proteins

a proteins.AGPs.AGP

cell wall.deg

cell wall.degradation.mannan-xylose-arabinose-fucose
cell wall.degradation.pectate lyases and polygalacturonases

S-assimilation
S-assimilation.APR

stress biotic PR proteins

tress.biotic.PR-proteins.PR6 (proteinase inhibitors)

miscellaneous

idases - copper flavone etc

DNA.synthesis/chromatin structure.histone.core.H3

protein.degradation

protein.degradation.ubiquitin
protein.degradation.ubiquitin.proteasome b)

fluorescence $(A U)$

200

C)

Choroplastic DCFDA

fluorescence (AU)

400

$100 \quad 200$

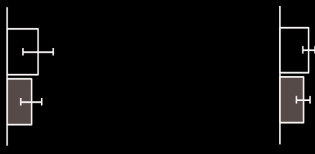

$\square$ WT

$\square$ Stpfld252
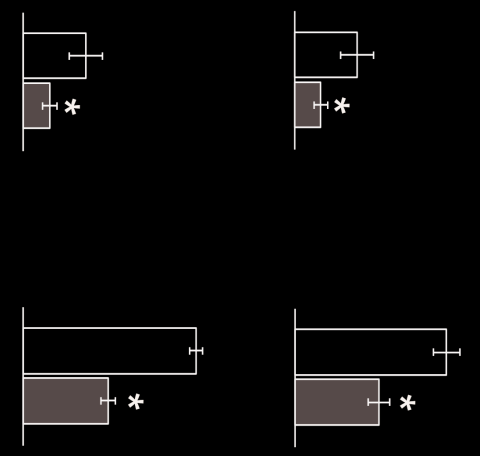

Fld-repressed genes

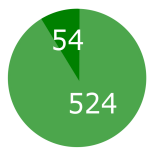

$\square \mathrm{FC}<0.25 \quad 0.25<\mathrm{FC}<0.5$

minor $\mathrm{CHO}$ metabolism.trehalose
minor CHO metabolism. trehalose.potential TPS/TPP

$\mathrm{N}$-metabolism

N-metabolism.nitrate metabolism

amino acid metabolism.degradation

metal handling

hormone metabolism

hormone metabolism.ethylene

hormone metabolism.ethylene.signal transduction

RNA.regulation of transcription

2/EREBP

RNA.regulation of transcription. $\mathrm{C} 3 \mathrm{H}$ zinc finger family

not assigned.no ontology.BTB/POZ domain-containing protein 


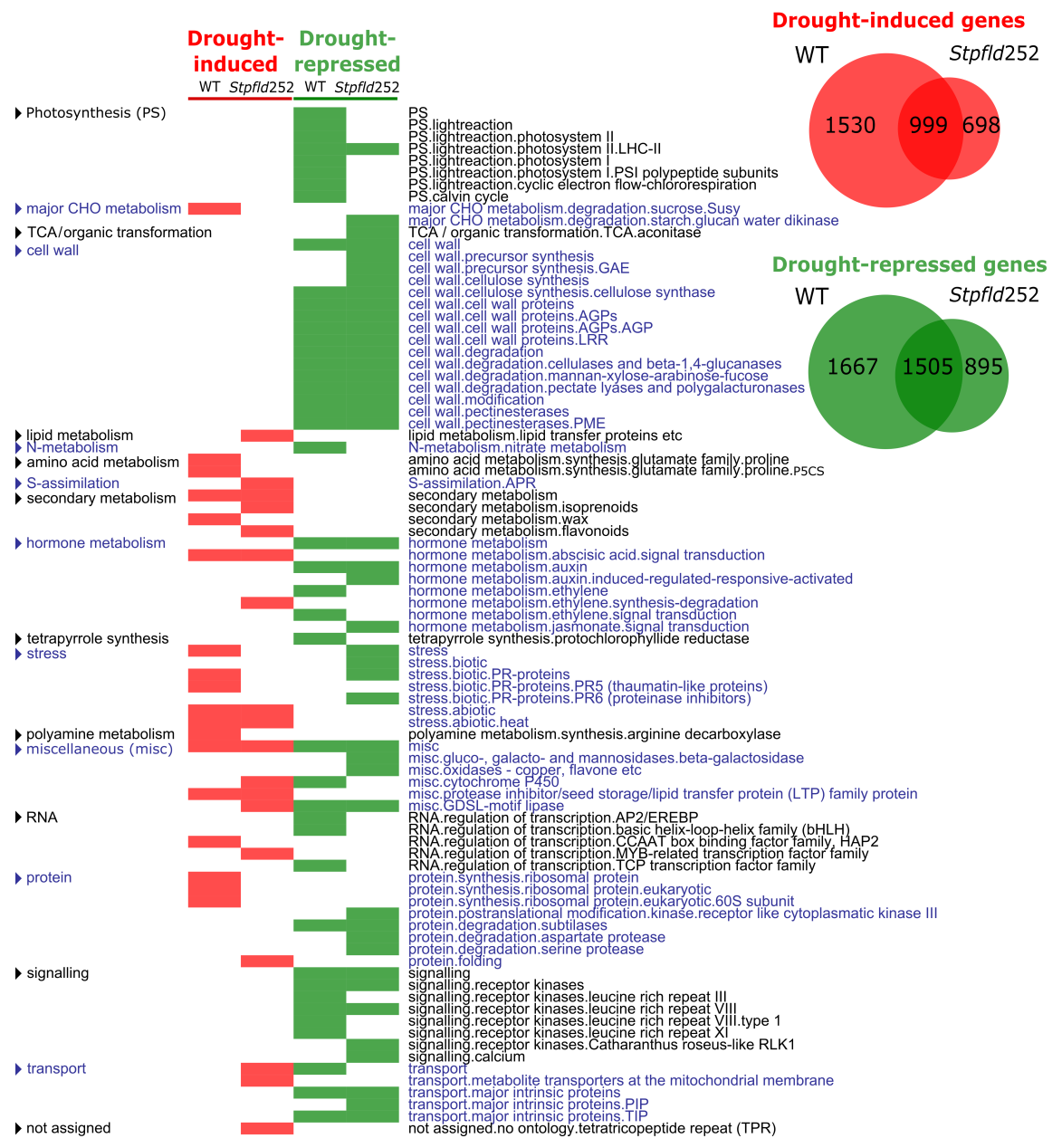




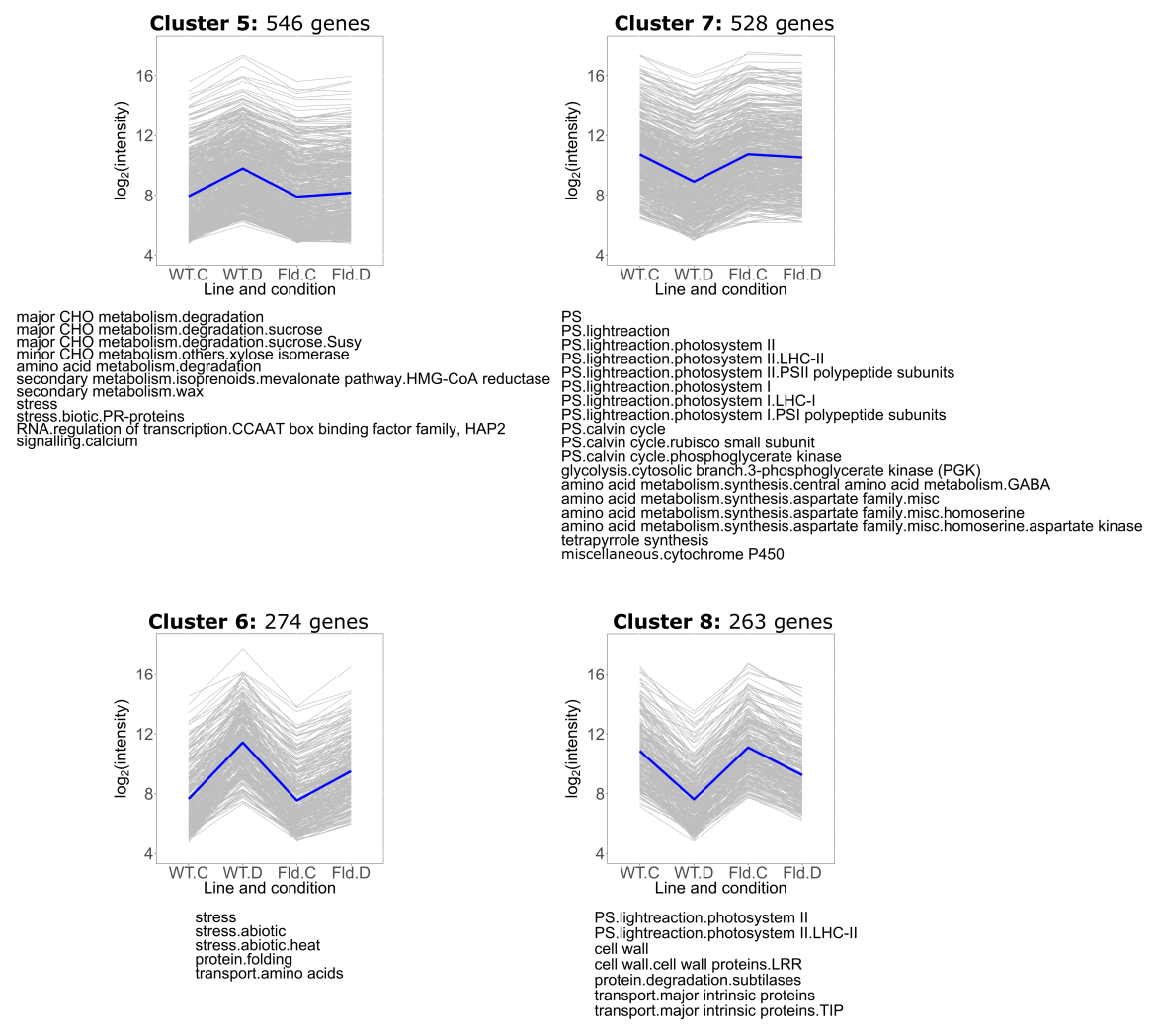




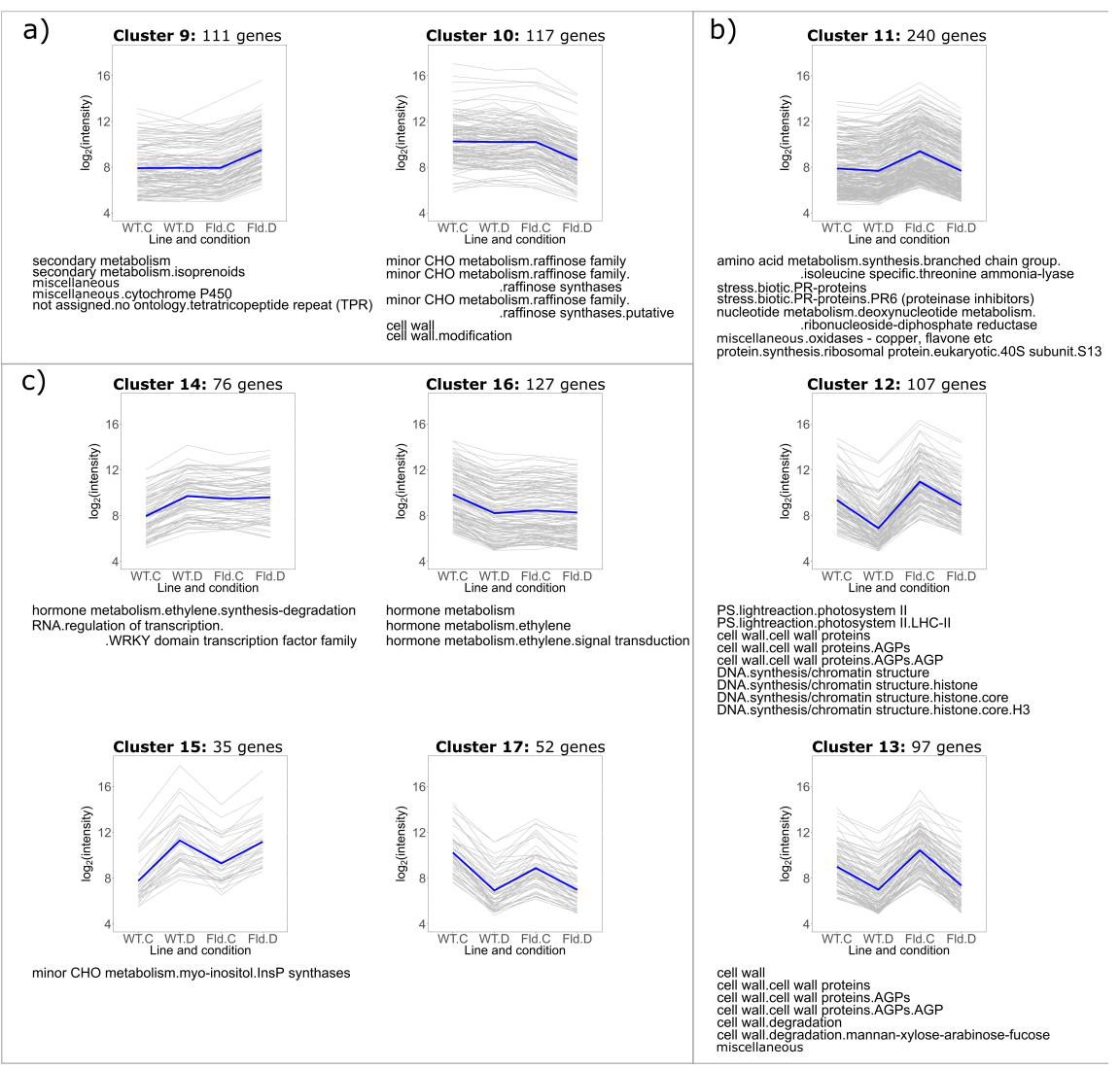




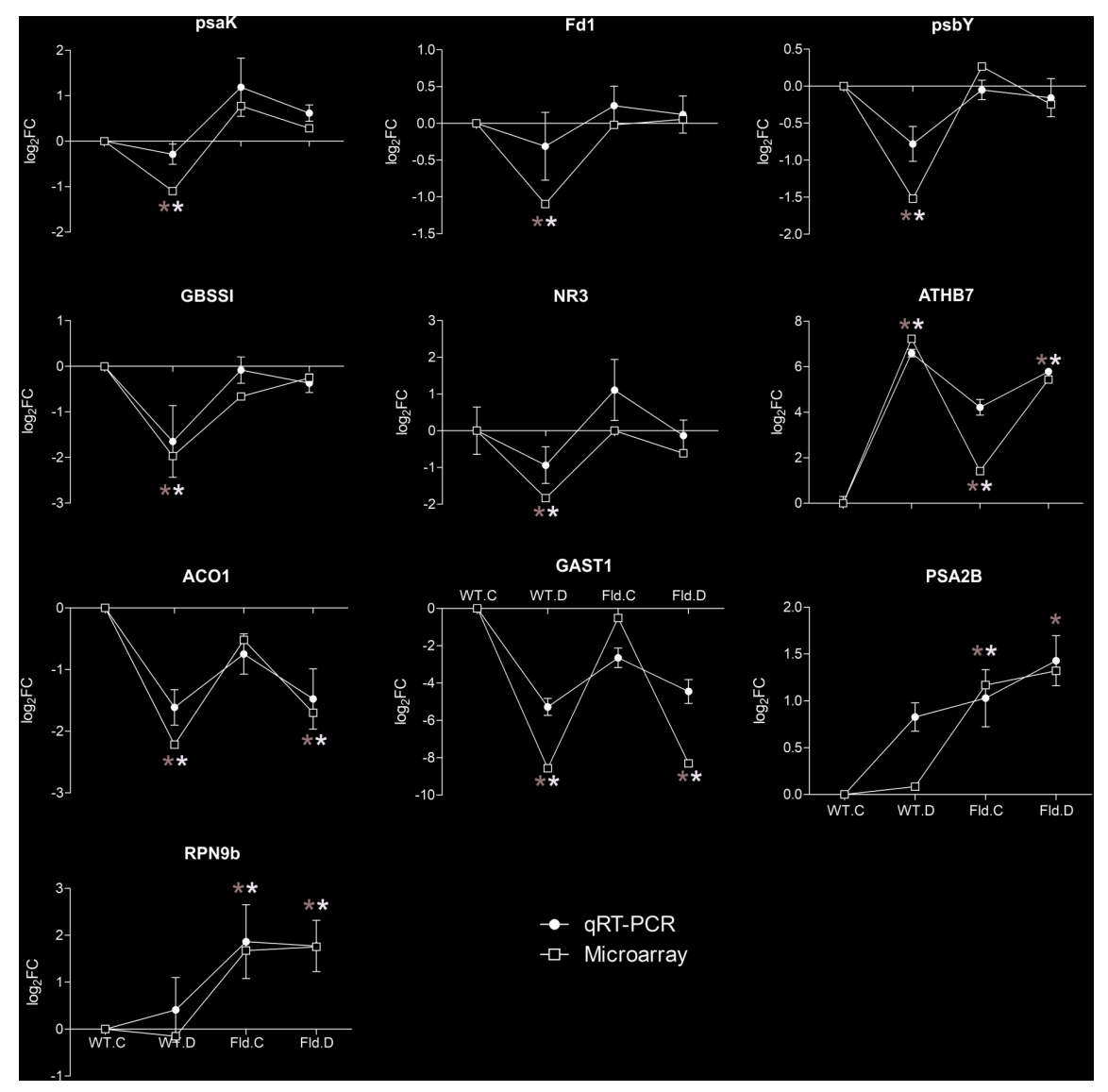


a)

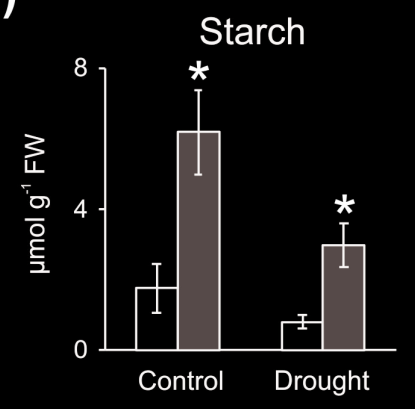

Fru

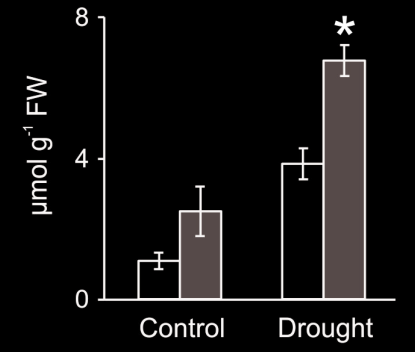

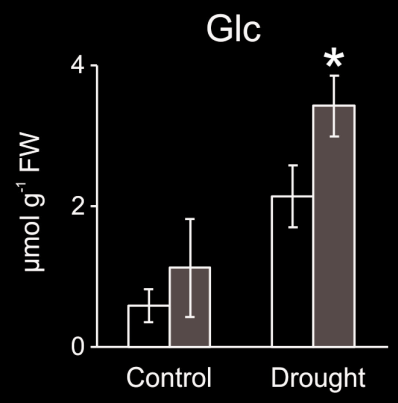

Suc

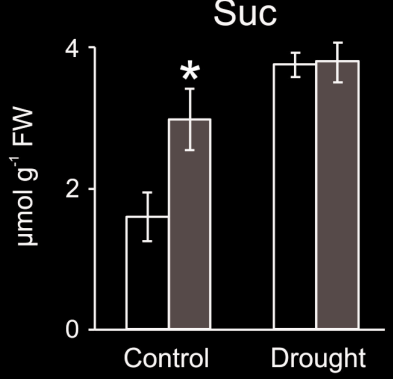

口WT

$\square$ Stpfld252

b)
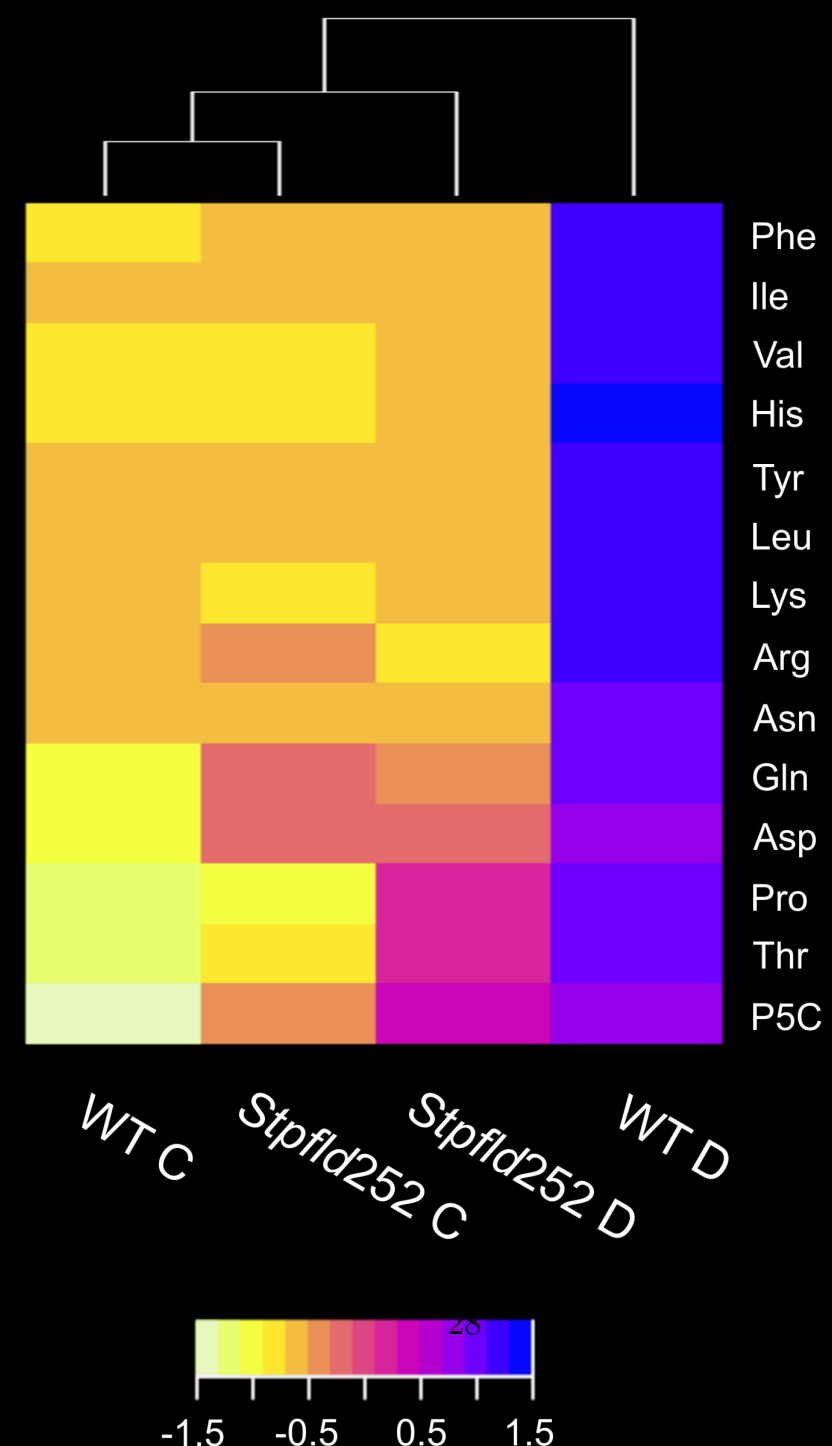


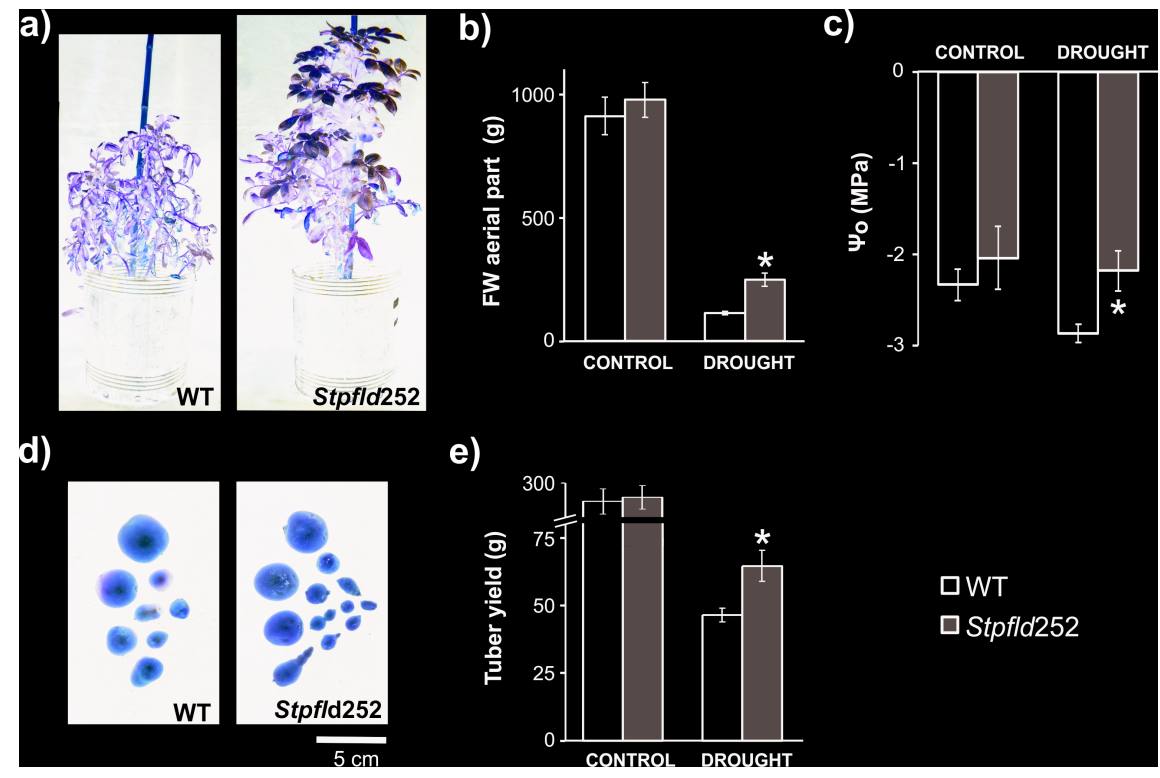

